\title{
Transition Density Estimates for Brownian Motion on Affine Nested Fractals
}

\author{
Pat J. Fitzsimmons ${ }^{1}$, Ben M. Hambly ${ }^{2}$, Takashi Kumagai ${ }^{3}$ \\ 1 Department of Mathematics, University of California, San Diego, La Jolla, CA 92093, USA \\ 2 Department of Mathematics and Statistics, University of Edinburgh, Mayfield Road, \\ Edinburgh, EH93JZ, UK \\ ${ }^{3}$ Department of Mathematics, Osaka University, Toyonaka 560, Japan
}

Received: 27 September 1993/in revised form: 14 December 1993

\begin{abstract}
A class of affine nested fractals is introduced which have different scale factors for different similitudes but still have the symmetry assumptions of nested fractals. For these fractals estimates on the transition density for the Brownian motion are obtained using the associated Dirichlet form. An upper bound for the diagonal can be found using a Nash-type inequality, then probabilistic techniques are used to obtain the off-diagonal bound. The approach differs from previous treatments as it uses only the Dirichlet form and no estimates on the resolvent. The bounds obtained are expressed in terms of an intrinsic metric on the fractal.
\end{abstract}

\section{Introduction}

The study of diffusion processes on finitely ramified fractals has seen the development of probabilistic techniques which can be used to study the heat equation on such fractals. The initial work was done on the Sierpinski gasket [5], in which the existence of a Brownian motion, its uniqueness with respect to the local symmetries of the set and estimates on the heat kernel were obtained. An important property needed was the finite ramification of the fractal, that any part of the fractal can be disconnected by the removal of only a finite number of points. The existence of a Brownian motion on a class of fractals with this property, called nested fractals, was shown in [27]. As yet the uniqueness of the process has only been proved for a subset of these fractals, [2] and only some properties are known [21, 23, 27]. There has also been some work on the Sierpinski carpet, an infinitely ramified fractal, in which the existence of a Brownian motion has been demonstrated [3] and estimates obtained on the heat kernel [4]. More general infinitely ramified fractals have been considered in [26], though all must have the property that the spectral dimension is less than two. Recently the extension to fractals in which the spectral dimension is greater than two has been accomplished by Barlow and Bass. For a review of the physics literature in this area see [13].

Another approach has been to discuss analysis on fractals directly [16], where Laplacians are constructed on Sierpinski gaskets and [17] for a large class of 
finitely ramified fractals, P.C.F. self-similar sets, which includes nested fractals. These ideas enable Laplacians to be constructed as limits of difference operators on the discrete lattices which approximate the fractal. They also allow the Dirichlet form on the fractal to be described as the limit of Dirichlet forms on the lattices.

In this paper we will consider a class of finitely ramified fractals, more general than nested fractals, which we call affine nested fractals. They have the property that the similitudes used can have different contraction ratios but they must still preserve the symmetry of the fractal. In the same manner as for nested fractals [12, 25] we can construct a Laplacian on the fractal via a Dirichlet form. There is a natural measure, suggested by [20], for this Laplacian and our aim will be to obtain heat kernel bounds for the transition semigroup with respect to this measure.

It is also possible to define an infinite version of the fractal, by inverting one of the contractions and iterating. The Dirichlet form can be extended to this fractal and our estimates will be obtained for the heat kernel on this infinite fractal. There is a natural intrinsic metric for a Dirichlet form and we will prove the existence of a version of this metric on the fractal. In the case of finitely ramified fractals it can be thought of as a constant power of the effective electrical resistance between two points in the fractal. The metric also takes account of the shortest path between points. Our results will be presented in terms of this metric.

In order to obtain estimates on the heat kernel we establish a Nash inequality for the functions in the Dirichlet space. By [6] this provides a uniform upper bound on the decay of the transition semigroup. In [6] it is shown how the method of Davies (for more discussion see [8]), for obtaining off diagonal bounds for the semigroup can be used for general Dirichlet forms with the Nash inequality. This method cannot be applied in this context due to the singularity of the energy forms with respect to the Hausdorff measure on the fractal [24]. Instead we turn to the probabilistic techniques developed for nested fractals in [23].

The lower bound can be obtained from the Dirichlet form wihtout using probabilistic estimates. This approach requires the Hölder continuity of the transition density. This is obtained via the scaling in the Dirichlet form and control of the continuity of the functions in the Dirichlet space by the Dirichlet norm. A chaining argument can then be used to extend the result form a small ball about the diagonal to the whole space. We prove the lower bound for the unbounded fractal where there is an exact scaling, and then use an inequality for the reflected process to obtain the bound for the compact fractal.

The Bernoulli measure we use for the discussion of the heat kernel on the fractal is the measure $\mu$ based on the electrical resistance. The dimension of the fractal based on this measure is the similarity dimension, $S$, (see $[10,20])$. The spectral dimension can then be expressed in terms of this dimension as $d_{s}=2 S /(S+1)$. The walk dimension $d_{w}$ will be computed by determining the shortest path metric on the fractal. In our case there is a relation between the Hausdorff dimension $d_{f}$ (with respect to the shortest path metric), $d_{w}$ and $d_{s} ; d_{s}=2 d_{f} / d_{w}$. This relationship has been shown to hold in other cases $([4,5,23])$.

The fundamental scaling factors for the fractals will be denoted as $\mu$ for the mass, $\tau$ for the time, $\rho$ for the conductance and $\alpha$ for the spatial scaling.

The main result is stated for the infinite affine nested fractal $\hat{E}$, in the following theorem, 


\section{Theorem 1.1.}

(1) There exists a jointly continuous transition density $\hat{p}_{t}(x, y)$ for the semigroup on the fractal $\hat{E}$.

(2) There exist constants $c_{5.9}, c_{5.10}, c_{6.1}, c_{6.2}$ such that $c_{6.1} t^{-d_{s} / 2} \exp \left(-c_{6.2} \Psi(d(x, y), t)\right) \leqq \hat{p}_{t}(x, y) \leqq c_{5.9} t^{-d_{s} / 2} \exp \left(-c_{5.10} \Psi(d(x, y), t)\right)$, for all $0<t<\infty, x, y \in \hat{E}$, where

$$
\Psi(z, t)=\left(z^{d_{w}} t^{-1}\right)^{1 /\left(d_{w}-1\right)} .
$$

In this paper, $c_{i}(i \in \mathbf{N})$ will be used as a positive finite constant whose value remains fixed within each proof and depends only on the affine nested fractal in question, while $c_{n, i}(i \in \mathbf{N})$ denotes a fixed constant which appears in section $n$.

The work in Sects. 3, 5 and 6 was done by the third author while visiting the university of Cambridge with the support of the Japan Association for Mathematical Sciences and Trinity College. The work in Sects. 4 and 6 of this paper was done while the second author was visiting the University of California, San Diego with the help of NSF grant DMS 8657483 and at the University of Cambridge with the support of the UK SERC.

\section{Affine Nested Fractals and Their Dirichlet Forms}

In [27], Lindstrøm introduced a class of finitely ramified fractals called nested fractals. The Dirichlet forms for these fractals were constructed by Kusuoka [25] and Fukushima [11]. We begin by defining affine nested fractals which is a wider class of fractals and stating some results which will be needed subsequently.

Let $\alpha>1$, then an $\alpha$-similitude is a map $\Psi: \mathbf{R}^{D} \rightarrow \mathbf{R}^{D}$ such that

$$
\Psi(x)=\alpha^{-1} U(x)+a,
$$

where $U$ is a unitary, linear map and $a \in \mathbf{R}^{D}$. We will denote a finite family of $\alpha_{i}$-similitudes by $\Psi=\left\{\Psi_{1}, \ldots, \Psi_{N}\right\}$. Then for a set $A \subset \mathbf{R}^{D}$, define

$$
\begin{aligned}
\Phi(A) & =\bigcap_{i=1}^{N} \Psi_{i}(A), \\
\Phi_{n}(A) & =\Phi \circ \cdots \circ \Phi(A) .
\end{aligned}
$$

By Hutchinson [14], this mapping has a fixed point which is the unique compact set $E$ such that $E=\Phi(E)$ and this is a self-similar fractal.

As each $\Psi_{i}$ is a contraction, it has a unique fixed point. Let $F$ be the set of fixed points of the $\Psi_{i}$ 's, $1 \leqq i \leqq N$. A point $x \in F$ is called an essential fixed point if there exist, $i, j \in\{1, \ldots, N\}, i \neq j$ and $y \in F$ such that $\Psi_{i}(x)=\Psi_{j}(y)$. We write $F^{(0)}$ for the set of essential fixed points. Now define

$$
\Psi_{i_{1} \ldots i_{n}}(A)=\Psi_{t_{1}} \circ \cdots \circ \Psi_{i_{n}}(A) \quad A \subset \mathbf{R}^{D} .
$$

We will call the set $\Psi_{i_{1}, \ldots, i_{n}}\left(F^{(0)}\right)$ an $n$-cell and $\Psi_{i_{1}, \ldots, i_{n}}(E)$ an $n$-complex. The set of $n$-th iteration of $F^{(0)}$ can be defined as

$$
F^{(n)}=\bigcup_{i_{1}, \ldots, i_{n}=1}^{N} \Psi_{i_{1}, \ldots, i_{n}}\left(F^{(0)}\right) .
$$


The set $E$ can be recovered from the essential fixed points by setting

$$
F^{(\infty)}=\bigcup_{n=0}^{\infty} F^{(n)}, \quad E=\operatorname{cl}\left(F^{(\infty)}\right) .
$$

We can now define an affine nested fractal as follows.

Definition 2.1 The set $E$ is an affine nested fractal if $\left\{\Psi_{1}, \ldots, \Psi_{N}\right\}$ satisfy:

(A1) (connectivity). For any 1-cells $C$ and $C^{\prime}$, there is a sequence $\left\{C_{i}: i=0, \ldots, n\right\}$ $(n \in \mathbf{N})$ of 1-cells such that $C_{0}=C, C_{n}=C^{\prime}$ and $C_{i-1} \cap C_{i} \neq \phi, i=1, \ldots, n$.

(A2) (symmetry). If $x, y \in F^{(0)}$ then reflection in the hyperplane $H_{x y}=$ $\{z:|z-x|=|z-y|\}$ maps $F^{(n)}$ to itself.

(A3) (nesting). If $\left\{i_{1}, \ldots, i_{n}\right\},\left\{j_{1}, \ldots, j_{n}\right\}$ are distinct sequences then

$$
\Psi_{i_{1}, \ldots, i_{n}}(E) \cap \Psi_{j_{1}, \ldots, j_{n}}(E)=\Psi_{i_{1}, \ldots, i_{n}}\left(F^{(0)}\right) \cap \Psi_{j_{1}, \ldots, j_{n}}\left(F^{(0)}\right) .
$$

(A4) (open set condition). There is a non-empty, bounded, open set $V$ such that the $\Psi_{i}(V)$ are disjoint and $\bigcup_{i=1}^{N} \Psi_{i}(V) \subset V$.

Note that the difference between affine nested fractals and nested fractals is that the contraction rate for each similitude can be different for the former but not for the latter.

We briefly list up geometrical properties of affine nested fractals. Results (1), (2), (3) can be obtained exactly by the same proofs as for nested fractals [27], while (4) is noted by J. Murai [28].

\section{Proposition 2.2.}

(1) If $x, y, x^{\prime}, y^{\prime} \in F^{(0)}$ and $|x-y|=\left|x^{\prime}-y^{\prime}\right|$, then there is a symmetry $U$ (i.e. reflection in $(A 2))$ such that $U(x)=x^{\prime}$ and $U(y)=y^{\prime}$.

(2) Set $l_{1}=\min \left\{|x-y| ; x, y \in F^{(0)}, x \neq y\right\}$. Then, for $x, y \in F^{(0)}$, there is a strict 1 -walk $s_{1}, \ldots, s_{n}$ (i.e. $s_{i}$ and $s_{i+1}$ join in the same $1-\operatorname{cell}\left(\Psi_{j(i)}\right.$, say) and $\left|s_{i}-s_{i+1}\right|=\alpha_{j(i)}^{-1} l_{1}$ for $1 \leqq i \leqq n-1)$ such that $s_{1}=x, s_{n}=y$ and $s_{k} \in F^{(1)}-F^{(0)}, 2 \leqq k \leqq n-1$.

(3) Any 1-cell contains at most one element of $F^{(0)}$.

(4) $\Psi_{i}(E) \cap \Psi_{j}(E)(i \neq j)$ is at most one point for $1 \leqq i, j \leqq N$.

The key ingredient in constructing a process on an affine nested fractal is to determine a sequence of random walks with suitably nested transition probabilities. On nested fractals, this has been studied in [27]. We define edge equivalence classes so that

$$
\left\{|x-y| ; x, y \in F^{(0)}, x \neq y\right\}=\left\{l_{1}, \ldots, l_{s_{0}}\right\}, \quad 0<l_{1}<\cdots<l_{s_{\circ}},
$$

and define $m_{s}=\#\left\{y \in F^{(0)}:|x-y|=l_{s}\right\}$. For each $x \in F^{(m)}$, let $N_{m}^{i}(x)$ be the set of $F^{(m)}$-neighbors $y$ of $x$ such that $|x-y|=\left(\alpha_{i_{1}} \ldots \alpha_{i_{m}}\right)^{-1} l_{i}$, where $x, y \in \Psi_{i_{1}}, \ldots, i_{m}(E)$ for some $1 \leqq i_{1}, \ldots, i_{m} \leqq N$. Also, let $N_{m}(x)=\bigcup_{i=1}^{s_{0}} N_{m}^{i}(x)$. We call $(x, y) \in$ $F^{(0)} \times F^{(0)}$ type $i$ and write Type $(x, y)=i$ if $x$ and $y$ are $F^{(0)}$-neighbors and $|x-y|=$ $l_{i}$. Now we can define the space of possible transition probabilities for the discrete chains with a natural ordering

$$
\mathscr{P}=\left\{\left(p_{1}, \ldots, p_{s_{0}}\right): p_{1}>\cdots>p_{s_{0}}>0, \sum_{i=1}^{s_{0}} m_{i} p_{i}=1\right\} .
$$




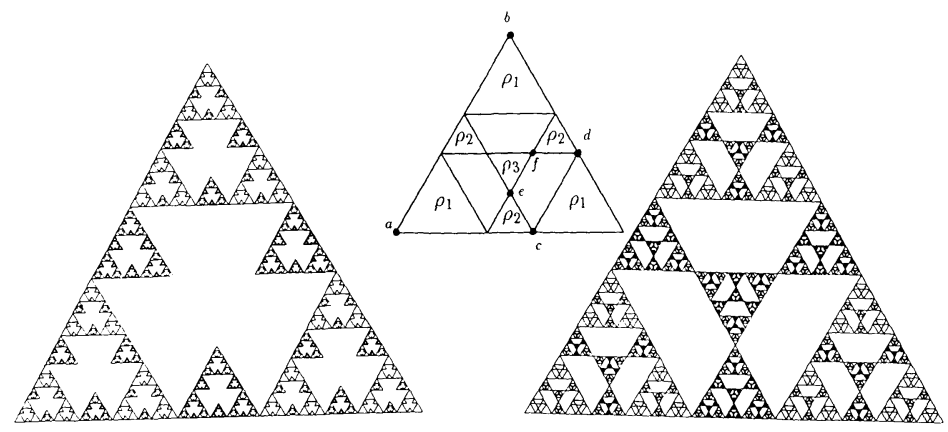

Fig. 1. Examples of affine nested fractals

We next define a size equivalence class. The sets $\Psi_{i}(E)$ and $\Psi_{j}(E)$ are the same size if they can be mapped to each other by the composition of the reflection maps which appear in $(A 2)$. Let the number of 1-complexes with different size be $k_{0}$. We can order the 1-cells by their size and put a weight on each size;

$$
\tilde{\mathbf{r}}=\left(\tilde{r}_{1}, \ldots, \tilde{r}_{k_{0}}\right), \quad \tilde{r}_{i}>0\left(1 \leqq i \leqq k_{0}\right) .
$$

In the following of this paper, we put $\tilde{e}$ (super tilde) when we distinguish cells by their size.

We call $(x, y) \in F^{(1)} \times F^{(1)}(x \neq y)$ size $i$ and write $\operatorname{Size}(x, y)=i$ if $x$ and $y$ are $F^{(1)}$-neighbors and the size of the 1-complex containing both $x$ and $y$ is $i$ (by Proposition 2.2 (4), we see that there is a unique 1-complex containing both $x$ and $y$ ).

These can then be used to construct a random walk on $F^{(1)}$. Let $X^{1}$ be a Markov chain on $F^{(1)}$ moving according to a set of basic transition probabilities $P \in \mathscr{P}$ divided by the weights $\tilde{\mathbf{r}}$; for $F^{(1)}$-neighbors $x, y(x \neq y)$, define

$$
p(x, y)=\frac{p_{\text {Type }(x, y)} \eta(x)}{\tilde{r}_{\text {Size }(x, y)}},
$$

where $\eta(x)$ is the normalization constant so that $\sum_{y \in N_{1}(x)} p(x, y)=1$. Otherwise $p(x, y)=0$.

The Markov chain on $F^{(1)}$ induces a Markov chain $X^{0}$ on $F^{(0)}$ by considering $X^{1}$ stopped when it hits $F^{(0)}$. Let $T_{0}^{0}\left(X^{1}\right)=\inf \left\{n \geqq 0: X_{n}^{1} \in F^{(0)}\right\}$, and $T_{i+1}^{0}=$ $\inf \left\{n>T_{i}^{0}\left(X^{1}\right): X_{n}^{1} \in F^{(0)}-\left\{X_{T_{1}^{0}\left(X^{1}\right)}^{1}\right\}\right.$ inductively for $i \geqq 0$. Then we require that $X_{T_{i}^{\circ}\left(X^{1}\right)}^{1}$ and $X_{i}^{0}$ have the same law. This corresponds to a fixed point for the transition probabilities whose existence was proved in [27] in the case of nested fractals. The following proposition can be proved in the same way as the corresponding part of [27].

Proposition 2.3. For any choice of $\tilde{\mathbf{r}}=\left(\tilde{r}_{1}, \ldots, \tilde{r}_{k_{0}}\right), \quad \tilde{r}_{i}>0 \quad\left(1 \leqq i \leqq k_{0}\right)$, there exists $\mathbf{p} \in \mathscr{P}$ such that the corresponding Markov chain $X_{T_{i}^{0}\left(X^{1}\right)}^{1}$ and $X_{i}^{0}$ have the same law.

In the following, we assume, without loss of generality, that the size of the 1-complex which contains an element of $F^{(0)}$ is $\tilde{r}_{1}$. Also, let $r_{i}=\tilde{r}_{\text {Size }\left(\Psi_{l}(E)\right)}$, where $\operatorname{Size}\left(\Psi_{i}(E)\right)$ is the size of $\Psi_{i}(E)(1 \leqq i \leqq N)$. Let $c=P^{x}\left(X_{\sigma\left(F^{(0)}\right)}^{1}=x\right)$ for $x \in F^{(0)}$, where 
$\sigma\left(F^{(0)}\right)=\inf \left\{n>0: X_{n}^{1} \in F^{(0)}\right\}$, then the conductance for the 1-complex containing an element of $F^{(0)}$ can be expressed as

$$
\rho_{1}=\frac{1}{1-c},
$$

which is independent of $x$ (see [25] and Theorem 5.2 of [22]). If we set $\lambda=\rho_{1} r_{1}$, then the conductance for $\Psi_{i}(E)$ is $\rho_{i}=\lambda / r_{i}$.

Throughout this paper, we assume the following:

Assumption 2.4. For all $1 \leqq i \leqq N, \rho_{i}>1$.

For $x, y \in F^{(0)}$ with $x \neq y$ set $\Pi_{x y}=p_{s}$ if $|x-y|=l_{s}$. Also, let $\mu$ be a Bernoulli measure on $E$ such that $\mu\left(\Psi_{i}(E)\right)=\mu_{i}>0\left(\sum_{i=1}^{N} \mu_{i}=1\right)$. We can now define the Dirichlet form for the affine nested fractal in the same way as [17] and [25]. Let $f$, $g \in l\left(F^{(\infty)}\right)=\left\{f: F^{(\infty)} \rightarrow \mathbf{R}\right\}$ and define

$$
\begin{aligned}
\mathscr{E}_{n}(f, g)= & \frac{1}{2} \sum_{1 \leqq k_{1} \ldots k_{n} \leqq N} \sum_{x, y \in F^{(0)}} \rho_{k_{1}, \ldots, k_{n}}\left(f\left(\Psi_{k_{1}, \ldots, k_{n}}(x)\right)-f\left(\Psi_{k_{1}, \ldots, k_{n}}(y)\right)\right) \\
& \times\left(g\left(\Psi_{k_{1}, \ldots, k_{n}}(x)\right)-g\left(\Psi_{k_{1}, \ldots, k_{n}}(y)\right)\right) \Pi_{x y}
\end{aligned}
$$

where $\rho_{k_{1}}, \ldots, k_{n}=\rho_{k_{1}}, \ldots, \rho_{k_{n}}$. Then, from [25] and for the more general class of P.C.F. self-similar sets in [17], the sequence of forms is monotone so that we have the following.

Theorem 2.5. Let $f \in l\left(F^{(\infty)}\right)$,

$$
\mathscr{F}=\left\{f: \sup _{n} \mathscr{E}_{n}(f, f)<\infty\right\}, \quad \mathscr{E}(f, f)=\lim _{n \rightarrow \infty} \mathscr{E}_{n}(f, f) .
$$

1) Any function in $\mathscr{F}$ can be extended uniquely to a continuous function on $E$ (thus we can consider $\mathscr{F} \subset C(E)=\{f: f$ is a continuous function on $E\})$.

2) $(\mathscr{E}, \mathscr{F})$ is a local regular Dirichlet form on $\mathbf{L}^{2}(E, \mu)$ which has the following scaling property:

$$
\mathscr{E}(f, g)=\sum_{i=1}^{N} \rho_{i} \mathscr{E}\left(f \circ \Psi_{i}, g \circ \Psi_{i}\right) \text { for all } f, g \in \mathscr{F}
$$

3) If we set $\mathscr{E}_{\beta}(\cdot, \cdot)=\mathscr{E}(\cdot, \cdot)+\beta(\cdot, \cdot)_{\mathbf{L}^{2}(E, \mu)}$ for $\beta>0$, then $\mathscr{E}_{\beta}$ admits a positive symmetric continuous reproducing kernel $g_{\beta}^{E}(\cdot, \cdot)$.

Our interest here is to obtain estimates on the heat kernel for the corresponding diffusion process. For this purpose, we need a "natural" measure, which is suggested by the following theorem in [20].

\section{Theorem 2.6.}

1) Let $\Delta_{\mu}$ be the generator for $(\mathscr{E}, \mathscr{F})$ on $\mathbf{L}^{2}(E, \mu)$ and let $n^{\mu}(x)=\#\{\lambda \mid \lambda$ be an eigenvalue of $-\Delta_{\mu} \leqq x$. $\}$. Then, for the unique positive number $d_{s}(\mu)$ satisfying $\sum_{i=1}^{N}\left(\mu_{i} / \rho_{i}\right)^{d_{s}(\mu) / 2}=1$, the following inequality holds:

$$
0<\liminf _{x \rightarrow \infty} n^{\mu}(x) / x^{d_{s}(\mu) / 2} \leqq \limsup _{x \rightarrow \infty} n^{\mu}(x) / x^{d_{s}(\mu) / 2}<\infty .
$$


2) Let $S$ be the unique constant which satisfies $\sum_{i=1}^{N} \rho_{i}^{-S}=1$. Then,

$$
\max \left\{d_{s}(\mu): \mu \text { is a Bernoulli measure on } E .\right\}=\frac{2 S}{S+1}\left(\equiv d_{s}\right),
$$

where the maximum is attained only at the Bernoulli measure $\mu$ satisfying

$$
\mu_{i}=\rho_{i}^{-S} \text { for } 1 \leqq i \leqq N .
$$

In the following we define $\mu_{i}=\rho_{i}^{-S}$ for $1 \leqq i \leqq N$ and consider this measure unless otherwise stated.

Next we will consider unbounded affine nested fractals and their Dirichlet forms. In [12] and [31], such forms are used to identify spectral dimensions, which express the asymptotic frequency of the eigenvalues of the corresponding Laplace operators on nested fractals. Assume, without loss of generality, that $\Psi_{1}(x)=\alpha_{1}^{-1} x$. Let $E^{<n>}=\alpha_{1}^{n} E$ and $\hat{E}=\bigcup_{n=1}^{\infty} E^{<n>}$.

Set $F_{n}=\alpha_{1}^{n} F^{(n)}$. We change the definition of $F^{(n)}$ as follows: $F^{(0)}=\bigcup_{n=0}^{\infty} F_{n}$ and $F^{(n)}=\alpha_{1}^{-n} F^{(0)}$ for $n \in \mathbf{Z}$. We next define the address of an $n$-complex on $\hat{E}$. First, set

$$
\begin{aligned}
& \Omega_{0}=\left\{\omega \in\{0, \ldots, N\}^{\mathbf{Z}} \mid \exists \eta, \xi \in \mathbf{Z} \text { such that } \omega_{k}=1 \text { for all } k \leqq \xi,\right. \\
& \left.w_{k^{\prime}}=0 \text { for all } k^{\prime}>\eta \text { and } \omega_{k^{\prime \prime}} \neq 0 \text { for all } k^{\prime \prime} \leqq \eta\right\} .
\end{aligned}
$$

For $\omega \in \Omega_{0}$, define

$$
\begin{aligned}
& \eta_{0}(\omega)=\min \left\{k: \omega_{k}=0\right\}-1, \\
& \xi_{0}(\omega)=\max \left\{k: \omega_{j}=1 \forall j \leqq k\right\},
\end{aligned}
$$

(we just use $\eta_{0}, \xi_{0}$ when there is no confusion).

For an $n$-complex $D_{n} \subset \hat{E}$, we say the address of $D_{n}$ is $\omega \in \Omega_{0}$ if $\Psi_{1}^{\left(-\xi_{0}\right)}\left(D_{n}\right) \subset E$ and $D_{n}=\Psi_{1}^{\left(\xi_{0}\right) \circ \Psi_{\omega_{1+\xi_{0}}} \ldots \omega_{\eta_{0}}}(E)$. Here $\Psi_{1}^{(l)}$ is the $l$-th composition of $\Psi_{1}$ and we regard $\Psi_{\omega_{1+\xi_{0}} \ldots \omega_{\eta_{0}}}=I$ (identity map) if $\eta_{0}=\xi_{0}$. Thus from the definition we see that $\eta_{0}=n$ and that $\xi_{0}=-\min \left\{l \mid \Psi_{1}^{(l)}\left(D_{n}\right) \subset E\right\}$. For $\omega \in \Omega_{0}$, we define

$$
\hat{\Psi}_{\omega}(E) \equiv \Psi_{1}^{\left(\xi_{0}\right)} \circ \Psi_{\omega_{1+\xi_{0}} \ldots \omega_{\eta_{0}}}(E),
$$

which is the corresponding $\eta_{0}$-complex.

Next, define $\sigma_{n}: l\left(E^{<n>}\right) \rightarrow l(E)$ by

$$
\sigma_{n} f(x)=f\left(\alpha_{1}^{n} x\right)=f \circ \Psi_{1}^{(-n)}(x) \text { for } x \in E .
$$

Set $\mathscr{F}_{E^{<1>}}=\sigma_{-l} \mathscr{F}$ and

$$
\mathscr{E}_{E^{<l>}}(f, g)=\rho_{1}^{-l} \mathscr{E}\left(\sigma_{l} f, \sigma_{l} g\right) \text { for } f, g \in \mathscr{F}_{E^{<l>}} .
$$

Also, let $\tilde{\mu}$ be the Bernoulli measure on $\hat{E}$ such that

$$
\tilde{\mu}\left(E^{<n>}\right)=\mu_{1}^{-n}, \quad \tilde{\mu}\left(\hat{\Psi}_{\omega}(E)\right)=\mu_{1}^{\xi_{0}} \mu_{\omega_{1+\xi_{0}} \ldots \omega_{\eta_{0}}}
$$

for all $n, m \in \mathbf{N}, \omega \in \Omega_{0}$. Note that for the following theorem, $\mu_{i}$ could be any positive number such that $\sum_{i=1}^{N} \mu_{i}=1$.

Now we define a quadratic form on $\hat{E}$. Let

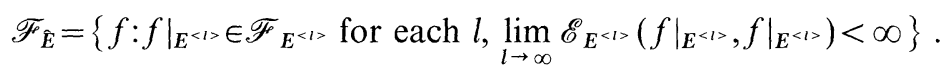


Then $\mathscr{F}_{\tilde{E}} \subset C(\hat{E})$. Set

$$
\hat{\mathscr{F}}=\widehat{\mathscr{F}}_{\hat{E}} \cap \mathbf{L}^{2}(\hat{E} ; \tilde{\mu}), \mathscr{E}(f, g)=\lim _{l \rightarrow \infty} \mathscr{E}_{E^{<>>}}\left(\left.f\right|_{E^{<l>}},\left.g\right|_{E^{<>>}}\right) \text {for } f, g \in \hat{\mathscr{F}} .
$$

Then we have the following results in th same way as [12].

Theorem 2.7. 1) $(\widehat{\mathscr{E}}, \hat{\mathscr{F}})$ is a local regular Dirichlet form on $\mathbf{L}^{2}(\widehat{E} ; \tilde{\mu})$ which has the following scaling property:

$$
\widehat{\mathscr{E}}(f, g)=\rho_{1} \widehat{\mathscr{E}}\left(f \circ \Psi_{1}, g \circ \Psi_{1}\right) \text { for all } f, g \in \hat{\mathscr{F}} .
$$

2) $(\widehat{\mathscr{E}})_{\beta}$ admits a positive symmetric continuous reproducing kernel $g_{\beta}(\cdot, \cdot)$.

We now deduce some results which will be used later. Set $\tau_{i}=\rho_{i} / \mu_{i}=\rho_{i}^{1+S}$.

Proposition 2.8. For the reproducing kernel $g_{\beta}(x, y)(\beta>0)$ associated with the Hilbert space $\left(\widehat{\mathscr{E}}_{\beta}, \hat{\mathscr{F}}\right)$, the following scaling property holds:

$$
g_{\beta}(x, y)=\rho_{1}^{-n} g_{\tau_{1}^{-n} \beta}\left(\alpha_{1}^{n} x, \alpha_{1}^{n} y\right), \quad \forall x, y \in \hat{E}, n \in \mathbf{N} .
$$

Proof. From the scaling (2.5) we can deduce the scaling for the form $\left(\widehat{\mathscr{E}}_{\beta}, \widehat{\mathscr{F}}\right)$,

$$
\begin{aligned}
\widehat{\mathscr{E}}_{\beta}(f, g) & =\widehat{\mathscr{E}}(f, g)+\beta(f, g) \\
& \left.=\rho_{1}^{n} \widehat{\mathscr{E}}\left(f \circ \Psi_{1}^{(n)}, g \circ \Psi_{1}^{(n)}\right)+\beta \mu_{1}^{n}\left(f \circ \Psi_{1}^{(n)}, g \circ \Psi_{1}^{(n)}\right)\right) \\
& =\rho_{1}^{n} \widehat{\mathscr{E}}_{\beta / \tau_{1}^{n}}\left(f \circ \Psi_{1}^{(n)}, g \circ \Psi_{1}^{(n)}\right)
\end{aligned}
$$

as desired. Recall the reproducing kernel property,

$$
\widehat{\mathscr{E}}_{\beta}\left(g_{\beta}(x, \cdot), f\right)=f(x), \quad \forall x \in \hat{E}, \forall f \in \hat{\mathscr{F}} .
$$

Now using this and (2.6)

$$
\begin{aligned}
g_{\beta / \tau_{1}}^{n}\left(\alpha_{1}^{n} x, y\right) & =\widehat{\mathscr{E}}_{\beta}\left(g_{\beta}(\cdot, x), g_{\beta / \tau_{1}^{n}}\left(\alpha_{1}^{n} \cdot, y\right)\right) \\
& =\dot{\rho}_{1}^{n} \widehat{\mathscr{E}}_{\beta / \tau_{1}^{n}}\left(\sigma_{-n} g_{\beta}(\cdot, \mathrm{x}), \sigma_{-n} g_{\beta / \tau_{1}^{n}}\left(\alpha_{1}^{n} \cdot, y\right)\right) \\
& =\rho_{1}^{n} \hat{\mathscr{E}}_{\beta / \tau_{1}^{n}}\left(g_{\beta / \tau_{1}^{n} \beta}(\cdot, y), g_{\beta}\left(\alpha_{1}^{-n} \cdot, x\right)\right) \\
& =\rho_{1}^{n} g_{\beta}\left(\alpha_{1}^{-n} y, x\right) .
\end{aligned}
$$

Then rearranging using symmetry and change of variables gives the result.

As we have the continuous reproducing kernel for each $x, y$, by [9] Lemma 2.1, the transition function possesses a kernel which satisfies the Chapman-Kolmogorov equations for all $x, y$ :

\section{Lemma 2.9.}

1) The transition function on $\hat{E}$ possesses a kernel $\hat{p}_{t}(x, y)$, so that $\hat{P}(t, x, d y)=\hat{p}_{t}(x, y) \tilde{\mu}(d y)$, and such that $\hat{p}_{t}(x, y)$ satisfies the Chapman-Kolmogorov equations,

$$
\hat{p}_{t}(x, y)=\int_{\widehat{E}} \hat{p}_{s}(x, z) \hat{p}_{t-s}(z, y) \mu(d z), \quad \forall x, y \in \hat{E} .
$$

2) The transition function on $E$ possesses a kernel $p_{t}^{E}(x, y)$, so that $P(t, x, d y)=p_{t}^{E}(x, y) \mu(d y)$, and such that $p_{t}^{E}(x, y)$ satisfies the Chapman-Kolmogorov equations. 
Properties of the Dirichlet form include the following Sobolev type inequality. Let $\rho_{i_{0}}=\min _{1 \leqq i \leqq N} \rho_{i}$ and consider the compact fractal.

Proposition 2.10. There exists a constant $c_{2.1}>0$ such that for all $f \in \mathscr{F}$ with $f(x)=0$ for some $x \in F^{(0)} \cap E$

$$
\|f\|_{\infty}^{2} \leqq c_{2.1} \mathscr{E}(f, f),
$$

where $\|\cdot\|_{\infty}$ is the supermum norm on $E$. There exist constants $c_{2.2}, c_{2.3}>0$ such that

$$
\begin{aligned}
\sup _{x, y \in E}|f(x)-f(y)| & \leqq c_{2.2} \mathscr{E}(f, f)^{1 / 2}, \quad \forall f \in \mathscr{F}, \\
\|f\|_{\infty}^{2} & \leqq c_{2.3}\left(\mathscr{E}(f, f)+\|f\|_{2}^{2}\right), \quad \forall f \in \mathscr{F} .
\end{aligned}
$$

Proof. Let $\bar{u}$ denote a 0 -harmonic function (an $i$-harmonic function is a function which is harmonic inside each $i$-complex) vanishing at some point $q$ of $F^{(0)}$. As $\Pi_{x y}>0\left(x \neq y, x, y \in F^{(0)} \cap E\right)$,

$$
\begin{aligned}
\mathscr{E}(\bar{u}, \tilde{u}) & =\frac{1}{2} \sum_{x, y \in F^{(0)}}(\bar{u}(x)-\bar{u}(y))^{2} \Pi_{x y} \\
& \geqq \frac{1}{2}\left(\max _{x \neq y} \Pi_{x y}\right) \sum_{p \in F^{(0)}} \bar{u}\left(p^{2}\right) \geqq c_{1}\|\bar{u}\|_{\infty}^{2},
\end{aligned}
$$

for some $c_{1}>0$. We use the maximum principle in the last inequality. Now by [20] Proposition 1.9 for $u \in \mathscr{F}$ and $\bar{u}$ the harmonic function with the same boundary values as $u$,

$$
\|u-\bar{u}\|_{\infty} \leqq c_{2} \mathscr{E}(u, u)^{1 / 2}
$$

so that

$$
\begin{aligned}
\|u\|_{\infty} & \leqq u-\bar{u}\left\|_{\infty}+\right\| \bar{u} \|_{\infty} \\
& \leqq c_{2} \mathscr{E}(u, u)^{1 / 2}+c_{1} \mathscr{E}(\bar{u}, \bar{u})^{1 / 2} \\
& \leqq\left(c_{1}+c_{2}\right) \mathscr{E}(u, u)^{1 / 2}
\end{aligned}
$$

by minimality of the harmonic functions. For (2.9) observe that $u(x)=f(x)-f(q)$ is a function in $\mathscr{F}$ vanishing at $q$, then from (2.8),

$$
|f(x)-f(y)| \leqq|f(x)-f(q)|+|f(q)-f(y)| \leqq 2\|u\|_{\infty} \leqq 2\left(c_{1}+c_{2}\right) \mathscr{E}(u, u)^{1 / 2},
$$

and then take the supremum.

For (2.10), note that as the set of 0 -harmonic functions is finite dimensional, the norms $\|\cdot\|_{\infty}$ and $\|\cdot\|_{2}$ are mutually equivalent on the space. Thus, using (2.11).

$$
\begin{aligned}
\|f\|_{\infty} & \leqq\|f-\bar{f}\|_{\infty}+\|\bar{f}\|_{\infty} \\
& \leqq c_{2} \mathscr{E}(f, f)^{1 / 2}+c_{3}\|\bar{f}\|_{2} \\
& \leqq c_{2} \mathscr{E}(f, f)^{1 / 2}+c_{3}\left(\|f\|_{2}+\|f-\bar{f}\|_{\infty}\right) \\
& \leqq c_{2.3}\left(\mathscr{E}(f, f)^{1 / 2}+\|f\|_{2}\right) .
\end{aligned}
$$

Corollary 2.11. Let $D_{n}$ be an $n$-complex such that $D_{n}=\hat{\Psi}_{\omega}(E)$ for $\omega \in \Omega_{0}$. Then

$$
\sup _{x, y \in D_{n}}|f(x)-f(y)| \leqq c_{2.2}\left(\rho_{1}^{\xi_{0}} \rho_{\omega_{1+\xi_{0}} \ldots \omega_{\eta_{0}}}\right)^{-1 / 2} \widehat{\mathscr{E}}(f, f)^{1 / 2} \text {. }
$$


Proof. From (2.9) and scaling we have that for the compact fractal

$$
\sup _{x, y \in E_{k_{1},}, . k_{n}}|f(x)-f(y)| \leqq c_{2.2} \rho_{k_{1}, \ldots, k_{n}}^{-1 / 2} \mathscr{E}(f, f)^{1 / 2} .
$$

Now, as $\Psi_{1}^{\left(-\xi_{0}\right)}\left(D_{n}\right) \subset E$,

$$
\begin{aligned}
\sup _{x, y \in D_{n}}|f(x)-f(y)| & =\sup _{x, y \in \Psi_{\rho_{\omega_{1}+\xi_{0}} \ldots \omega_{\eta_{0}}}(E)}\left|\sigma_{-\xi_{0}} f(x)-\sigma_{-\xi_{0}} f(y)\right| \\
& \leqq c_{2.2}\left(\rho_{\omega_{1+\xi_{0}} \ldots \omega_{\eta_{0}}}\right)^{-1 / 2} \mathscr{E}\left(\sigma_{-\xi_{0}} f, \sigma_{-\xi_{0}} f\right)^{1 / 2} \\
& =c_{2.2}\left(\rho_{1}^{\xi_{0}} \rho_{\omega_{1+\xi_{0}} \ldots \omega_{\eta_{0}}}\right)^{-1 / 2} \mathscr{E}_{E^{<-\xi_{0}>}}(f, f)^{1 / 2} .
\end{aligned}
$$

\section{Distance on Affine Nested Fractals}

In this section we introduce an intrinsic metric for the Dirichlet forms constructed in Sect.2. The distance on the wider class of fractals, P.C.F. self-similar sets, is discussed in [18] and [19]. Our distance is similar to that of [18].

We will first construct a distance on $E$. For $x, y \in F^{(m)} \cap E$, let

$$
\begin{gathered}
\pi_{m}(x, y)=\left\{\pi_{m}: \pi_{m} \text { is an } m \text {-walk in } E \text { from } x \text { to } y\right. \text { which does not } \\
\text { contain multiple points }\} .
\end{gathered}
$$

Here $\pi_{m}=\left\{p_{k}, p_{k+1}\right\}_{k=1}^{l}$ is called an $m$-walk if $l \in \mathbf{N}, p_{k} \in F^{(m)}$ for $1 \leqq k \leqq l, p_{k}$ and $p_{k+1}$ join in the same $m$-cell for $1 \leqq k \leqq l-1$. For $\pi_{m}=\left\{p_{k}, p_{k+1}\right\}_{k=1}^{l} \in \pi_{m}(x, y)$, we say the length of $\pi_{m}$ is $l$ and denote it by $\left|\pi_{m}\right|=l$. For $\pi \in \pi_{1}(x, y)$, let $\mathbf{v}_{k}(\pi)$ be an $s_{0^{-}}$ dimensional vector such that $\left(\mathbf{v}_{k}(\pi)\right)_{j}$ is the number of $k$-size $j$-type steps in the path $\pi\left(1 \leqq j \leqq s_{0}, 1 \leqq k \leqq k_{0}\right)$. Now, for a positive number $t$, set

$$
S_{t}^{i}=\left\{\sum_{k=1}^{k_{0}} \tilde{\rho}_{k}^{-t} \mathbf{v}_{k}(\pi) \mid \pi \in \pi_{1}(x, y), x, y \in F^{(0)} \cap E, y \in N_{0}^{i}(x)\right\},
$$

where $\tilde{\rho}_{k}=\lambda / \tilde{r}_{k}$.

In order to construct a distance, we must first solve an eigenvalue problem.

Proposition 3.1. Set

$$
\left(G_{t}(x)\right)_{i}=\max _{\mathbf{a} \in S_{t}^{i}} \mathbf{a} \cdot \mathbf{x} .
$$

Then, there exist $0<\gamma^{\prime}<S+1, \mathbf{x}>0$ such that $G_{\gamma^{\prime}}(\mathbf{x})=\mathbf{x}$.

This proposition is essentially the same as Proposition 3.4 of [23]. For the proof, we prepare a lemma.

Lemma 3.2. Let $B=\left\{\mathbf{x} \in \mathbf{R}^{s_{0}} \mid 0 \leqq x_{1} \leqq \cdots \leqq x_{s_{0}}\right\}$. Then $G_{t}(B) \subset B$.

Proof. Fix $p \in F^{(0)}, q \in N_{0}^{i}(p), q^{\prime} \in N_{0}^{i-1}(p)$. Let $U_{q q^{\prime}}$ be the reflection map which maps $q$ to $q^{\prime}$. Define $V=\left\{z \in \mathbf{R}^{D}:\left|z-q^{\prime}\right| \leqq|z-q|\right\}$. Also we define a map $T: \mathbf{R}^{D} \rightarrow \mathbf{R}^{D}$ by

$$
T z= \begin{cases}z & \text { if } z \in V \\ U_{q q^{\prime}} z & \text { otherwise. }\end{cases}
$$

For $\quad \mathbf{x} \in B$ and $i \geqq 2$, let $\left(G_{t}(\mathbf{x})\right)_{i}=\sum_{k=1}^{k_{0}} \tilde{\rho}_{k}^{-t} a_{1 k} x_{1}+\cdots+\sum_{k=1}^{k_{0}} \tilde{\rho}_{k}^{-t} a_{s 0 k} x_{r}$, $\left(\sum_{k=1}^{k_{0}} \tilde{\rho}_{k}^{-t} a_{1 k}, \ldots, \sum_{k=1}^{k_{0}} \tilde{\rho}_{k}^{-t} a_{s_{0} k}\right) \in S_{t}^{i}$. Then we know that there exists at least one 
1 -walk from $p$ to $q$ which has $k$-size $j$-type steps $a_{j k}$ times. Express the 1 -walk by $y_{0}, y_{1}, \ldots, y_{m}$, where $y_{0}=p, y_{m}=q$ and $m=\sum_{j, k} a_{j k}$.

Then we know Type $\left(T y_{i}, T y_{i+1}\right) \leqq \operatorname{Type}\left(y_{i}, y_{i+1}\right)$ because $\left|T y_{i}-T y_{i+1}\right| \leqq$ $\left|y_{i}-y_{i+1}\right|$ (where we set Type $\left(y_{i}, y_{i+1}\right)=0$ if $\left.y_{i}=y_{i+1}\right)$. Also, as the sizes of two 1-complexes are the same if they are mapped to each other reflections, $\operatorname{Size}\left(T y_{i}, T y_{i+1}\right)=\operatorname{Size}\left(y_{i}, y_{i+1}\right)$ when $T y_{i} \neq T y_{i+1}$. Denote $a_{j k}^{\prime}=\#\left\{\left(y_{i}, y_{i+1}\right)\right.$ : Type $\left.\left(T y_{i}, T y_{i+1}\right)=j, \operatorname{Size}\left(y_{i}, y_{i+1}\right)=k, 0 \leqq i \leqq m-1\right\}$. Then we have $\left(G_{t}(\mathbf{x})\right)_{i-1} \leqq$ $\sum_{j, k} \tilde{\rho}_{k}^{-t} a_{j k}^{\prime} x_{j} \leqq \sum_{j, k} \tilde{\rho}_{k}^{-t} a_{j k} x_{j}=\left(G_{t}(\mathbf{x})\right)_{i}$ because $\mathbf{x} \in B$.

Proof of Proposition 3.1. For fixed $t>0$, set

$$
K_{t}=\left\{A: A \text { is an } s_{0} \times s_{0} \text {-matrix, }(l \text {-th row of } A) \in S_{t}^{l}, 1 \leqq l \leqq s_{0}\right\},
$$

and $\lambda_{t}=\min _{A \in K_{t}}\{$ largest eigenvalue of $A\}$. We first prove that there exists $x_{t}>0$ such that $G_{t}\left(\mathbf{x}_{t}\right)=\lambda_{t} \mathbf{x}_{t}$.

Set $c_{0}=\min _{k} \tilde{\rho}_{k}^{-t}$. If $\mathbf{x} \in B$, then $\left(G_{t}(\mathbf{x})\right)_{1} \geqq c_{0} x_{1}$ and $\left(G_{t}(\mathbf{x})\right)_{i} \leqq c_{i} x_{1}$ for some $c_{i}>0\left(1 \leqq i \leqq s_{0}\right)$ because $\left(c_{i}, 0, \ldots, 0\right) \in S^{i}$ from Proposition $2.2(2)$. Thus, if $\mathbf{x} \in B$ and $x_{1}>0$, we know

$$
\frac{\left(G_{t}(\mathbf{x})\right)_{1}}{\sum_{i}\left(G_{t}(\mathbf{x})\right)_{i}} \geqq \frac{c_{0} x_{1}}{\sum c_{i} x_{1}}=\frac{c_{0}}{\sum c_{i}} \equiv \varepsilon
$$

Let $B_{\varepsilon}=\left\{x \in B: \sum x_{i}=1, x_{1} \geqq \varepsilon\right\}$ and $\tilde{G}_{t}(\mathbf{x})=\frac{1}{\sum\left(G_{t}(\mathbf{x})\right)_{i}} G_{t}(\mathbf{x})$ for $\mathbf{x} \in B_{\varepsilon}$. Then, by definition, $\left(\widetilde{G}_{t}(\mathbf{x})\right)_{1} \geqq \varepsilon$. Combining this with Lemma 3.2, we see $\widetilde{G}_{t}\left(B_{\varepsilon}\right) \subset B_{\varepsilon}$. Thus, by the fixed point theorem, there exists $\mathbf{x}_{t} \in B_{\varepsilon}$ such that $\widetilde{G}_{t}\left(\mathbf{x}_{t}\right)=\mathbf{x}_{t}$. If we define $\lambda_{t}^{\prime}=\sum\left(G_{t}\left(\mathbf{x}_{t}\right)\right)_{i}$, we have $G_{t}\left(\mathbf{x}_{t}\right)=\lambda_{t}^{\prime} \mathbf{x}_{t}$. By the Frobenius theorem, it is easy to deduce $\lambda_{t}=\lambda_{t}^{\prime}$. When $t=0, \lambda_{t}>1$ because $A \mathbf{1} \geqq \mathbf{1}$ for all $A \in K_{0}$. We next consider the case $t=S+1$. For $A \in K_{S+1}$ which attains the minimum largest eigenvalue, decompose it as $A=\sum_{k} \tilde{\rho}_{k}^{-(S+1)} A_{k}$, where $\left(A_{k}\right)_{i j}=a_{j k}^{i}$ if $(i$-th row of $A)=$ $\left(\sum_{k=1}^{k_{0}} \tilde{\rho}_{k}^{-\gamma^{\prime}} a_{1 k}^{i}, \ldots, \sum_{k=1}^{k_{0}} \tilde{\rho}_{k}^{-\gamma^{\prime}} a_{\text {sok }}^{i}\right)$. Clearly, $\max _{i} \sum_{j}\left(A_{k}\right)_{i j} \leqq($ number of $k$-size 1 complexes in $E$ ). Thus, $\max _{i} \sum_{j} A_{i j} \leqq \sum_{m=1}^{N} \rho_{m}^{-(S+1)}<\sum_{m=1}^{N} \rho_{m}^{-S}=1$ and hence $\lambda_{s+1}<1$. As $\lambda_{t}$ is a minimum of the largest eigenvalue of matrices in $K_{t}, \lambda_{t}$ moves continuously with respect to $t$. Thus there exists $0<\gamma^{\prime}<S+1$ such that $\lambda_{\gamma^{\prime}}=1$.

Remark 3.3. For $\pi, \pi^{\prime} \in \pi_{1}(x, y)$, we can write $\pi<\pi^{\prime}$ if $\mathbf{v}_{k}(\pi) \leqq \mathbf{v}_{k}\left(\pi^{\prime}\right)$ for all $1 \leqq k \leqq k_{0}$. By this $\pi_{1}(x, y)$ is an ordered set. Define $\hat{S}_{\gamma^{\prime}}^{i}=\left\{\pi \in S_{\gamma^{\prime}}^{i} \mid \pi\right.$ is a minimum with respect to the order. $\}$ Then, it is enough to consider $\hat{S}_{\gamma^{\prime}}^{i}$ when we calculate $G_{\gamma^{\prime}}(\mathbf{x})$.

In the case of Fig. $2, \hat{S}^{1}$ is as follows:

$$
\hat{S}^{1}=\left\{\left(2 \rho_{1}^{-\gamma^{\prime}}+\rho_{2}^{-\gamma^{\prime}}, 0\right),\left(\rho_{3}^{-\gamma^{\prime}}, 2 \rho_{1}^{-\gamma^{\prime}}\right),\left(2 \rho_{1}^{-\gamma^{\prime}}+\rho_{3}^{-\gamma^{\prime}}, \rho_{1}^{-\gamma^{\prime}}\right),\left(4 \rho_{1}^{-\gamma^{\prime}}+\rho_{3}^{-\gamma^{\prime}}, 0\right)\right\} .
$$

( $\hat{S}^{2}$ has many elements and we leave it to the reader to write them all out.)

Now we define the distance on $F^{(m)}$ as follows:

$$
d_{F^{(m)}}(x, y)=\min _{\pi \in \pi_{m}(x, y), \pi=\left\{\left(p_{k}, p_{k+1}\right)\right\}_{k=1}^{|\pi|}} \sum_{k=1}^{|\pi|} \rho_{\operatorname{Size}\left(p_{k}, p_{k+1}\right)}^{-\gamma^{\prime}} \mathbf{x}_{\text {Type }\left(p_{k}, p_{k+1}\right)} .
$$

Here $\mathbf{x}$ is the one which appeared in Proposition 3.1 and $\operatorname{Size}(p, q)=i_{1} \cdots i_{m}$ if the $m$-complex containing both $p$ and $q$ is $\Psi_{i_{1}, \ldots, i_{m}}(E)$. 

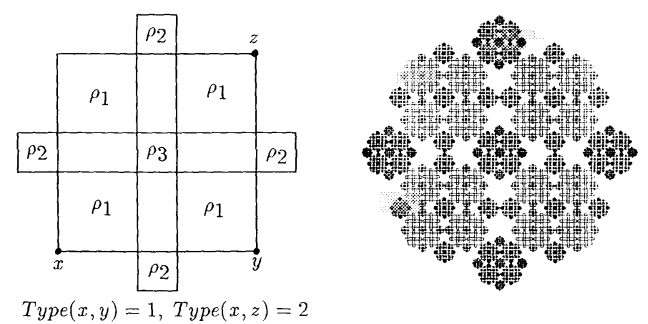

Fig. 2. An example of an affine nested fractal and its shortest path

\section{Lemma 3.4.}

1) If $p, q \in F^{(0)} \cap E$ and $\operatorname{Type}(p, q)=i$, then $d_{F^{(1)}}(p, q)=\left(\mathbf{e}_{i}, G_{\gamma^{\prime}}(\mathbf{x})\right)=x_{i}$, where $\mathbf{e}_{i}$ is a unit vector whose $i$-th component is 1 .

2) $d_{F^{(m)}}(x, y)=d_{F^{(m+1)}}(x, y)$ if $x, y \in F^{(m)} \cap E$.

Proof. 1) is clear by definition of $G_{\gamma^{\prime}}$.

For $p, q \in F^{(0)}$, let $\pi=\left\{p_{k}, p_{k+1}\right\}_{k=1}^{m}$ be a minimal $F^{(1)}$-path. Define the $F^{(m+1)}$ path between $\Psi_{\omega}(p), \Psi_{\omega}(q)\left(\omega \in\{1, \ldots, N\}^{m}\right)$ as

$$
\pi_{\omega}=\left\{\Psi_{\omega}\left(p_{k}\right), \Psi_{\omega}\left(p_{k+1}\right)\right\}_{k=1}^{m}
$$

To prove 2), pick a minimal $F^{(m+1)}$-path $\pi_{m+1}$ between $x$ and $y$ and induce a $F^{(m)}$-path $\pi_{m}=\left\{q_{k}, q_{k+1}\right\}_{k=1}^{n}$. Then, by the nesting property ((A3) in the Definition 2.1) of affine nested fractals, $\pi_{m+1}$ moves in the same $m$-complex ( $\Psi_{\omega}(E)$, say) between $q_{k}$ and $q_{k+1}$. It must be of the form of (3.5) as $\pi_{m+1}$ is minimal. By definition and 1), the $d_{F^{(m+1)}}$-distance of $\pi_{m+1}$ between $q_{k}$ and $q_{k+1}$ is

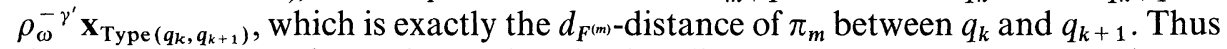

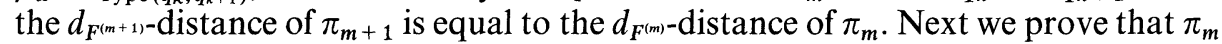
is a minimal $F^{(m)}$-path between $x$ and $y$. If it is not, we have a minimal $F^{(m)}$-path $\pi_{m}^{\prime}$ between $x$ and $y$. In which case we can construct an $F^{(m+1)}$-path $\pi_{m+1}^{\prime}$ from $\pi_{m}^{\prime}$ by putting (3.5) in each step. Then the $d_{F^{(m+1)}}$-distance of $\pi_{m+1}^{\prime}$ is equal to the $d_{F^{(m)}-\text { distance of } \pi_{m}^{\prime}}$ and this contradicts the minimal choice of $\pi_{m+1}$.

This lemma enables us to define a metric $d$ on $F^{(\infty)} \cap E$ by setting $d(p, q)=d_{F^{(m)}}(p, q)$ for $p, q \in F^{(m)} \in E$. We extend this metric to a metric on $E$ as follows.

Theorem 3.5. For any choice of $p, q \in E$, define $d(p, q)$ by

$$
d(p, q)=\lim _{n \rightarrow \infty} d\left(p_{n}, q_{n}\right)
$$

where $p_{n}, q_{n} \in F^{(\infty)} \cap E$ and $p_{n} \rightarrow p, q_{n} \rightarrow q$ as $n \rightarrow \infty$. Then $d$ is well defined and $d$ is a metric on $E$.

Proof. The following is the same proof as [18]. Note that by the nesting property ((A3) in the Definition 2.1) of affine nested fractals, $p_{n} \rightarrow p$ if and only if, for each $m$, there exists $n(m)$ such that, for all $n>n(m), p_{n} \in U_{m, p}$, where $U_{m, p}=\bigcup_{\omega: p \in \Psi_{\omega}(E),|\omega| \geqq m} \Psi_{\omega}(E)$. 
Now, let $p_{n} \rightarrow p, p_{n}^{\prime} \rightarrow p$ as $n \rightarrow \infty$, where $\left\{p_{n}\right\},\left\{p_{n}^{\prime}\right\} \subset F^{(\infty)}$. Note that for $\omega \in\{1, \ldots, N\}^{m}$

$$
d\left(\Psi_{\omega}(E)\right) \equiv \max _{x, y \in \Psi_{\omega}(E) \cap F^{(x)}} d(x, y) \leqq R^{m} M,
$$

where $R=\max _{i=1}^{N} \rho_{i}^{-\gamma^{\prime}}<1$ and $M=\max _{i} \mathbf{x}_{i}$. Hence, for sufficiently large $n$,

$$
d\left(p_{n}, p_{n}^{\prime}\right) \leqq \sum_{\omega \in U_{m, p}} d\left(\Psi_{\omega}(E)\right) \leqq C R^{m} M
$$

where $C=\# \bigcup_{i \neq j}\left(\Psi_{i}(E) \cap \Psi_{j}(E)\right)$. Therefore $d\left(p_{n}, p_{n}^{\prime}\right) \rightarrow 0$ as $n \rightarrow \infty$. By the triangle inequality,

$$
\left|d\left(p_{n}, q_{n}\right)-d\left(p_{m}, q_{m}\right)\right| \leqq d\left(p_{n}, p_{m}\right)+d\left(q_{n}, q_{m}\right) .
$$

Combining this with the above facts, we see that the limit of $d\left(p_{n}, q_{n}\right)$ exists as $n \rightarrow \infty$. The same discussion shows that $d$ is well defined, and it is clear that $d$ is a metric on $E$.

For $p, q \in E^{<n>}$, define

$$
d^{n}(p, q)=\rho_{1}^{n \gamma^{\prime}} d\left(\Psi_{1}^{(n)}(p), \Psi_{1}^{(n)}(q)\right) .
$$

Then $d^{n}$ is a metric on $E^{<n>}$. Further there exists an $N$ such that for each $m$,

$$
d^{m+N}(p, q)=d^{m+N+k}(p, q) \text { for all } p, q \in E^{<m>}, k \in \mathbf{N} \text {. }
$$

To prove this, define

$$
P^{(n)}=\Psi_{1}^{(n)}(E) \cap\left(\bigcup_{|\omega|=n, \omega \neq 1 \ldots 1} \Psi_{\omega}(E)\right) .
$$

First, the minimal $F^{(N)}$-path between any two distinct elements in $P^{(N)}$ does not exit $P^{(1)}$ for large $N$ between because otherwise the distance would be infinite as

$$
\min _{x \in P^{(n)}, y \in P^{(n+1)}} d(x, y)=\rho_{1} \min _{x \in P^{(n+1)}, y \in P^{(n+2)}} d(x, y) .
$$

Then, we easily see that the minimal path between any two elements of $\Psi_{1}^{(N)}(E)$ is contained in $\Psi_{1}(E)$, which gives (3.6).

By (3.6), we can define a metric on $\hat{E}$ which we will also denote $d(p, q)$. By suitable normalization of $\mathbf{x}$, we can set $d(E) \equiv \max _{p, q \in E} d(p, q)=1$. Clearly, this metric has the following scaling:

$$
d(p, q)=\rho_{1}^{\gamma^{\prime}} d\left(\Psi_{1}(p), \Psi_{1}(q)\right) \text { for all } p, q \in \hat{E} .
$$

Next we introduce a $\Lambda$-approximation to $\hat{E}$. For $n \in \mathbf{Z}$, set

$$
\Lambda_{n}=\left\{\omega \in \Omega_{0} \mid \rho_{1}^{\xi_{0}-1} \rho_{\omega_{\xi_{0}} \ldots \omega_{\eta_{0}-1}}<\rho_{i_{0}}^{n} \leqq \rho_{1}^{\xi_{0}-1} \rho_{\omega_{\xi_{0}} \ldots \omega_{\eta_{0}}}\right\}
$$

Here $\rho_{i_{0}}=\max _{1 \leqq i \leqq N} \rho_{i}$ and we set $\rho_{\omega_{\xi_{0}} \ldots \omega_{n_{0}-1}}=1$ when $\eta_{0}=\xi_{0}$ (an analogous set was considered in [20]). Define $H^{\left(\Lambda_{n}\right)}=\bigcup_{\omega \in \Lambda_{n}}^{\hat{\Psi}_{\omega}}\left(F^{(0)} \cap E\right)$. We can prove the following in the same way as [20] Lemma 2.6, which is an easy consequence of the definition,

$$
\begin{gathered}
\bigcup_{\omega \in \Lambda_{n}} \widehat{\Psi}_{\omega}(E)=\hat{E} \quad \text { for all } n \in \mathbf{Z}, \\
\hat{\Psi}_{\omega}(E) \cap \hat{\Psi}_{\omega^{\prime}}(E) \subset H^{\left(\Lambda_{n}\right)} \quad \text { for all } \omega, \omega^{\prime} \in \Lambda_{n}, \omega \neq \omega^{\prime} .
\end{gathered}
$$


Consider two points $p, q \in H^{\left(\Lambda_{n}\right)}$ which belong to $\widetilde{\Psi}_{\omega}(E)\left(\omega \in \Lambda_{n}\right)$. If the shortest path between $p, q$ is inside $\hat{\Psi}_{\omega}(E)$, then $d(p, q)=\left(\rho_{1}^{\xi_{0}-1} \rho_{\omega_{\xi_{0}} \ldots \omega_{\eta_{0}}}\right)^{-\gamma^{\prime}} \cdot \mathbf{x}_{\text {Type }(p, q)}$. Further, by the geometrical property of affine nested fractals, if the path between $p, q$ is not inside $\hat{\Psi}_{\omega}(E)$, then it passes through at least one element in $H^{\left(\Lambda_{n}\right)} \cap \hat{\Psi}_{\omega}(E)^{c}$.

Thus there exist constants $c_{3.2}>c_{3.1}>0$ (independent of $p, q, n$ ) such that for $p$, $q$ in a $H^{\left(\Lambda_{n}\right)}$-neighborhood,

$$
c_{3.1} \rho_{i_{0}}^{-n \gamma^{\prime}} \leqq d(p, q) \leqq c_{3.2} \rho_{i_{0}}^{-n \gamma^{\prime}} .
$$

Using this we have the following scaling property.

Proposition 3.6. There exists $c_{3.3}>0$ (independent of $p, q$ ) such that for all $p, q \in E$ and for all $\omega \in \bigcup_{m \in \mathbf{N}}\{1, \ldots, N\}^{m}$,

$$
c_{3.3} \rho_{w}^{-\gamma^{\prime}} d(p, q) \leqq d\left(\Psi_{w}(p), \Psi_{w}(q)\right) \leqq \rho_{w}^{-\gamma^{\prime}} d(p, q) .
$$

Proof. If the minimal path between $\Psi_{w}(p)$ and $\Psi_{w}(q)$ is inside $\Psi_{w}(E)$, then, by the construction of the metric, $d\left(\Psi_{w}(p), \Psi_{w}(q)\right)=\rho_{w}^{-\gamma^{\prime}} d(p, q)$. This gives the upper bound. To show the lower bound it is enough to consider the case when the minimal path is outside $\Psi_{w}(E)$. Choose $n$ such that

$$
\Psi_{\omega^{\prime}}(E) \subset \Psi_{\omega}(E) \subset \Psi_{\omega^{\prime \prime}}(E) \text { for some } \omega^{\prime} \in \Lambda_{n}, \omega^{\prime \prime} \in \Lambda_{n-1} .
$$

Then, there exists $c_{1}, c_{2}>0$ (independent of the choice of $\omega$ ) such that

$$
c_{1} \rho_{i_{0}}^{n} \leqq \rho_{w} \leqq c_{2} \rho_{i_{0}}^{n} .
$$

On the other hand, using (3.10), we have

$$
d\left(\Psi_{\omega}(p), \Psi_{\omega}(q)\right) \geqq c_{3.1} \rho_{i_{0}}^{-n \gamma^{\prime}} .
$$

Combining (3.11), (3.12) and using the fact that $d(p, q) \leqq 1$, we obtain the lower bound.

\section{Remark 3.7.}

1) In proposition 3.6, the equality does not hold in general. In fact, in the case of Fig. 1 in Sect. 2 , if $\rho_{1} \ll \rho_{2}, \rho_{3}$, then

$$
d(c, d)=d(c, e)+d(e, f)+d(f, d)<\rho_{1}^{-\gamma^{\prime}} d(a, b) .
$$

2) In [19], Kigami introduced a resistance metric $R_{\mathscr{L}}$ on $E$. Using Proposition 3.6 and the results in [19], we see that there exist $c_{3.4}, c_{3.5}>0$ such that

$$
c_{3.4} d(p, q) \leqq R_{\mathscr{L}}(p, q)^{\gamma^{\prime}} \leqq c_{3.5} d(p, q) \text { for all } p, q \in E .
$$

As the Hausdorff dimension of $E$ w.r.t. $R_{\mathscr{L}}$ is $S$ (see [19]), the Hausdorff dimension w.r.t. $d$ is $S / o^{\prime}$.

3) In the case of nested fractals, we have the following relation between the Euclidean metric and the distance studied in this section.

$$
c_{3.6} d(p, q) \leqq|p-q|^{d_{c}} \leqq c_{3.7} d(p, q) \quad \text { for all } p, q \in E,
$$

where $c_{3.6}, c_{3.7}$ are positive constants and $d_{c}$ is a chemical exponent studied in [1] and [23]. 


\section{A Nash Inequality for Affine Nested Fractals}

In this section we will establish a Nash-type inequality, and thereby a diagonal upper bound on the transition density for Brownian motion on the fractal. We will first prove the Nash inequality for the unbounded fractal $\hat{E}$ and then use an embedding argument to deduce the analogous inequality for $E$.

Theorem 4.1. There is a constant $c_{4.1}$ such that

$$
\|f\|_{2}^{2+4 / d_{s}} \leqq c_{4.1} \hat{\mathscr{E}}(f, f)\|f\|_{1}^{4 / d_{s}}
$$

for all $f \in \hat{\mathscr{F}} \cap L^{1}(\widehat{E}, \tilde{\mu})$.

Proof. For $f \in C_{b}(\hat{E})$, the set of continuous bounded functions on $\widehat{E}$, set

$$
U_{\beta}^{(\infty)} f(x)=E^{x}\left[\int_{0}^{\infty} e^{-\beta t} f\left(X_{t}\right) d t\right]=\int_{0}^{\infty} g_{\beta}(x, y) f(y) d \tilde{\mu}(y) .
$$

Let $f \in \hat{\mathscr{F}}$ and consider $\sup _{x \in D_{n}}|f(x)|^{2}$ for an $n$ complex $D_{n}=\widetilde{\Psi}_{\omega}(E), \omega \in \Omega_{0}$. As in Corollary 2.11 we can use scaling in (2.10) in order to obtain

$$
\sup _{x \in D_{n}}|f(x)|^{2} \leqq c_{2.3}\left(\left(\rho_{1}^{\xi_{0}} \rho_{\omega_{1+\xi_{0}} \ldots \omega_{\eta_{0}}}\right)^{-1} \mathscr{E}_{E^{<-\xi_{0}>}}(f, f)+\mu_{1}^{\xi_{0}} \mu_{\omega_{1+\xi_{0}} \ldots \omega_{\eta_{0}}}(f, f)_{E^{<-\xi_{0}>}}\right) .
$$

By choosing $\omega \in \Lambda_{0}$, the values of $\left(\rho_{1}^{\xi_{0}} \rho_{\omega_{1+\xi_{0}} \ldots \omega_{\eta_{0}}}\right)^{-1}$ and $\mu_{1}^{\xi_{0}} \mu_{\omega_{1+\xi_{0}} \ldots \omega_{\eta_{0}}}$ are within a constant of 1 . Thus we have that

$$
\sup _{x \in D_{n}}|f(x)|^{2} \leqq c_{1} \hat{\mathscr{E}}_{1}(f, f),
$$

where $c_{1}$ is independent of the choice of $\omega \in \Omega_{0}$. Therefore we have the extension of (2.10) to the unbounded fractal. Now, following the proof of Theorem 2.3 in [12], and using the extension of $(2.10)$

$$
\sup _{y \in D_{n}} \sqrt{g_{1}(y, y)} \leqq \sup _{y} \frac{\sup _{x \in D_{n}} g_{1}(x, y)}{\sqrt{g_{1}(y, y)}} \leqq \sup _{f \in \mathscr{F}} \frac{\sup _{x \in D_{n}}|f(x)|}{\sqrt{\hat{\mathscr{E}}_{1}(f, f)}} \leqq c_{1}<\infty .
$$

Thus $c_{2}=\left\|g_{1}\right\|_{\infty}<\infty$. Since

$$
\widehat{\mathscr{E}}(f, f)=\sup _{\beta>0} \beta\left(f, f-\beta U_{\beta}^{(\infty)} f\right)_{\mu} \geqq\left(f, f-U_{1}^{(\infty)} f\right)_{\mu}
$$

we have $\|f\|_{2}^{2} \leqq\left(f, U_{1}^{(\infty)} f\right)_{\mu}+\widehat{\mathscr{E}}(f, f)$, and so

$$
\|f\|_{2}^{2} \leqq c_{2}\|f\|_{1}^{2}+\widehat{\mathscr{E}}(f, f), \quad f \in \hat{\mathscr{F}} .
$$

Now notice that if $\tilde{f}(x)=f\left(\alpha_{1}^{n} x\right)$ where $n \in \mathbf{Z}$, then $\|\tilde{f}\|_{p}=\mu_{1}^{-n / p}\|f\|_{p}$, where $\mu_{1}=\rho_{1}^{-s}$ and $\hat{\mathscr{E}}(\tilde{f}, \tilde{f})=\rho_{1}^{-n} \hat{\mathscr{E}}(f, f)$. Replacing $f$ in (4.2) by $\tilde{f}$ and using the fact that $\mu_{1}=\rho_{1}^{-S}$ we obtain

$$
\|f\|_{2}^{2} \leqq \rho_{1}^{S n} c_{2}\|f\|_{1}^{2}+\rho_{1}^{-n(1+S)} \hat{\mathscr{E}}(f, f), \quad f \in \widehat{\mathscr{F}}, n \in \mathbf{Z} .
$$

Since $d_{s} / 2=S /(S+1),(4.3)$ yields immediately

$$
\|f\|_{2}^{2} \leqq R^{d_{s} / 2} c_{2}\|f\|_{1}^{2}+R^{-1} \hat{\mathscr{E}}(f, f), \quad f \in \hat{\mathscr{F}}, R>0 .
$$

Optimizing the choice of $R$ in (4.4) we obtain (4.1) with a constant $c_{4.1}$ depending only on $\left\|g_{1}\right\|_{\infty}$ and $d_{s}$. 
Remark 4.2. By the above proof, we can see that (4.1) holds if and only if the Bernoulli measure is $\mu_{1}=\rho_{1}^{-S}$.

The analogous Nash-type inequality for the compact fractal $E$ is a consequence of Theorem 3.1 and the following

Lemma 4.3. There is a linear map $J: \mathscr{F} \rightarrow \widehat{\mathscr{F}}$ and constants $c_{4.2}$ and $c_{4.3}(\delta)$ such that for all $f \in \mathscr{F}$,

(i) $J f=f$ on $E$;

(ii) If $f \in L^{p}(\hat{E}, \tilde{\mu})$, then $\|J f\|_{p} \leqq c_{4.2}\|f\|_{p}$, for all $1 \leqq p \leqq \infty$;

(iii) $\widehat{\mathscr{E}}(J f, J f) \leqq c_{4.3}(\delta)\left(\mathscr{E}(f, f)+\delta\|f\|_{2}^{2}\right)$, for all $\delta>0$.

Proof. Let $F^{\prime}$ denote the set of points $i \in F^{(0)} \cap E$ that belong to at least one 0 -complex of $\hat{E} \backslash E$. Given $i \in F^{\prime}$ let $C_{i}$ be the 1-complex of $E$ containing $i$, and let $C_{i, 1}, C_{i, 2}, \ldots, C_{i, n}$ be the 1-complexes of $\hat{E} \backslash E$ containing $i$. Let $\Psi_{i, j}$ denote the contraction which fixes $i$ and scales by $\alpha_{j}$. For each pair $i, j$ there is a uniquely determined reflection $R_{i, j}$ that maps $\Psi_{i, j}^{-1}\left(C_{i, j}\right)$ onto $C_{i}$ (and fixes $i$ ). Now given $f \in \mathscr{F}$ define $\tilde{f}$ on $\hat{E}$ by

$$
\tilde{f}(x)= \begin{cases}f(x), & x \in E ; \\ f\left(R_{i, j} \Psi_{i, j}^{-1}(x)\right), & x \in C_{i, j}, i \in F^{\prime} \\ 0, & \text { otherwise. }\end{cases}
$$

Finally, let $C_{i, j}^{\prime}$ denote the unique 2-complex contained in $C_{i, j}$ that contains $i$, and define a 2-harmonic function $h$ on $\hat{E}$ by requiring that $h=1$ on $E$ and that $h=0$ off $E \cup\left(\bigcup_{i, j} C_{i, j}^{\prime}\right)$. The desired extension of $f$ is $J f=\tilde{f} h$. It is easy to see that if $n$ is the maximum number of 1-complexes to which an element of $F^{\prime}$ can belong and $\bar{\mu}=\max _{i} \mu_{i}$, then

$$
\|J f\|_{p}^{p} \leqq n \bar{\mu}\|f\|_{p}^{p}, \quad 1 \leqq \forall p<\infty,
$$

and clearly $\|J f\|_{\infty} \leqq\|f\|_{\infty}$. Moreover, from the estimate ([11], Theorem 1.4.2(ii))

$$
\widehat{\mathscr{E}}(u v, u v) \leqq 2\left[\|u\|_{\infty}^{2} \hat{\mathscr{E}}(v, v)+\|v\|_{\infty}^{2} \hat{\mathscr{E}}(u, u)\right],
$$

and the local nature of $\widehat{\mathscr{E}}$, it follows easily that

$$
\widehat{\mathscr{E}}(J f, J f) \leqq \mathscr{E}(f, f)+2 n \bar{\mu}\left[\mathscr{E}(f, f)+c_{1}\|f\|_{\infty}^{2}\right],
$$

where $c_{1}=\widehat{\mathscr{E}}(h, h)$. But from $(2.10),\|f\|_{\infty}^{2} \leqq c_{2.3}\left(\mathscr{E}(f, f)+\|f\|_{2}^{2}\right)$. Together with (4.5) this yields point (iii) of the lemma.

Combining the lemma with Theorem 4.1 we arrive at the main result of this section.

Theorem 4.4. If $\delta>0$ then there is a constant $c_{4.3}(\delta)$ such that

$$
\|f\|_{2}^{2+4 / d_{s}} \leqq c_{4.3}(\delta)\left(\mathscr{E}(f, f)+\delta\|f\|_{2}^{2}\right)\|f\|_{1}^{4 / d_{s}} \quad \forall f \in \mathscr{F} \cap L^{1}(\hat{E}, \tilde{\mu}) .
$$

By Theorem 2.1 of [6] the Nash inequality of Theorem 4.4 is equivalent to an upper bound on $p_{t}^{E}(x, y)$ on the compact fractal for small $t$. For the transition density on the unbounded fractal $\hat{p}_{t}(x, y)$, Theorem 2.1 of [6] with Theorem 4.1 above gives an upper bound for all times: 
Theorem 4.5. For the transition density $p_{t}^{E}(x, y)$ of Brownian motion on the compact affine nested fractal $E$ there is a constant $c_{4.4}>0$ such that

$$
p_{t}^{E}(x, y) \leqq c_{4.4} t^{-d_{s} / 2} \quad \forall x, y \in E, \forall t \in(0,1] .
$$

For the transition density $\hat{p}_{t}(x, y)$, there is a constant $c_{4.5}>0$ such that

$$
\hat{p}_{t}(x, y) \leqq c_{4.5} t^{-d_{s} / 2}, \quad \forall x, y \in \hat{E}, \forall t>0 .
$$

Remark. The equivalence between the decay of the heat kernel and the Nash inequality has been used in [29] as a definition of the spectral dimension.

Using the upper bound estimate, we can show the joint continuity of the heat kernel.

Lemma 4.6. $\hat{p}_{t}(x, y)$ (resp. $\left.p_{t}^{E}(x, y)\right)$ is jointly continuous in $(t, s, y) \in(0, \infty) \times \hat{E} \times \hat{E}$ (resp. $(t, x, y) \in(0,1] \times E \times E)$.

Proof. As $\hat{\mathscr{F}} \subset C(\hat{E})$ and $\hat{P}_{t}: L^{2}(\mu) \rightarrow \widehat{\mathscr{F}}$, we see that $\hat{P}_{t} f \in C(\hat{E})$ for all $f \in L^{2}(\mu)$. Also by Chapman-Kolmogorov and symmetry

$$
\left\|\hat{p}_{t}(x, \cdot)\right\|_{2}^{2}=\int_{E}\left(\hat{p}_{t}(x, y)\right)^{2} \mu(d y)=\hat{p}_{2 t}(x, x)<\infty,
$$

so that $\hat{p}_{t}(x, \cdot) \in L^{2}(\mu)$. Hence, as

$$
\hat{p}_{t}(x, y)=\hat{P}_{t / 2}\left(\hat{p}_{t / 2}(\cdot, y)\right)(x)
$$

the map $x \mapsto \hat{p}_{t}(x, y)$ is continuous for each fixed $t$ and $y \in \hat{E}$. Now use Cauchy-Schwarz and the diagonal upper bound, to obtain

$$
\hat{p}_{t}(x, y) \leqq\left[\hat{p}_{t}(x, x) \hat{p}_{t}(y, y)\right]^{1 / 2} \leqq c_{1}(t), \quad \forall x, y \in \hat{E} .
$$

This estimate allows dominated convergence to be used in (4.7) to show that $(x, y) \mapsto \hat{p}_{t}(x, y)$ is continuous. The next step is to get joint continuity in $(t, x)$. By [7] Proposition 1.18, if $\hat{P}_{0}=I, \hat{P}_{t+s}=\hat{P}_{t} \hat{P}_{s}$ and $\lim _{t \downarrow 0} \hat{P}_{t} f=f$, for all $f \in \mathbf{L}^{2}(\hat{E} ; \tilde{\mu})$, then $(t, f) \mapsto \hat{P}_{t} f$ is jointly continuous. As these conditions hold for our strongly continuous contraction semigroup we can take $\left(t_{n}, y_{n}\right) \mapsto(t, y)$ then set $f_{n}=\hat{p}_{s}\left(\cdot, y_{n}\right)$, $\bar{t}_{n}=t_{n}-s$ for an $s$ such that $s<t_{n}$. Now by the above

$$
\hat{p}_{t_{n}}\left(\cdot, y_{n}\right)=\hat{P}_{\bar{t}_{n}} f_{n} \rightarrow \hat{P}_{t-s} f=\hat{p}_{t}(\cdot, y) .
$$

Thus the map $(t, y) \mapsto \hat{p}_{t}(x, y)$ is jointly continuous for each $x$.

To get the joint continuity in all three variables we use Champman-Kolmogorov again. Fix $s>0$ and let $t_{n} \rightarrow t>s, x_{n} \rightarrow x, y_{n} \rightarrow y$. Then by the continuity results so far

$$
\lim _{n \rightarrow \infty} \hat{p}_{s}\left(x_{n}, z\right) \hat{p}_{t_{n}-s}\left(z, y_{n}\right)=\hat{p}_{s}(x, z) \hat{p}_{t-s}(z, y),
$$

for each $z \in \hat{E}$. Now apply (4.8), to get

$$
\hat{p}_{s}\left(x_{n}, z\right) \hat{p}_{t_{n}-s}\left(z, y_{n}\right) \leqq c_{1}(s) \hat{p}_{s}\left(x_{n}, z\right),
$$

and as the family $\hat{p}_{s}\left(x_{n}, z\right)$ is integrable we can use dominated convergence in (2.7) to extend the continuity of $\hat{p}_{t}(x, y)$ to $\{(t, x, y): t>s, x, y \in \hat{E}\}$. Now let $s \downarrow 0$ to obtain the joint continuity on $\{(t, x, y): t>0, x, y \in \hat{E}\}$. The joint continuity of $p_{t}^{E}(x, y)$ is proved in the same way.

By this lemma and Proposition 2.8, we have the scaling property of $\hat{p}_{t}(x, y)$ : 
Proposition 4.7. For the transition density associated with the Laplacian defined by the Dirichlet form $(\hat{\mathscr{E}}, \hat{\mathscr{F}})$, the following scaling property holds:

$$
\hat{p}_{t}(x, y)=\mu_{1}^{-n} \hat{p}_{\tau_{1}^{n} t}\left(\alpha_{1}^{n} x, \alpha_{1}^{n} y\right), \quad \forall x, y \in \hat{E}, t>0, n \in \mathbf{N} .
$$

\section{Hitting Time Estimates and the Upper Bound Estimates of the Transition Density}

For $A \subset \widehat{E}$, let $\sigma(A)=\inf \{t \geqq 0: X(t) \in A\}$, where $X(t)$ is the diffusion process corresponding to $(\widehat{\mathscr{E}}, \hat{\mathscr{F}})$. Set

$$
W_{n}=\inf \left\{t \geqq 0: X(t) \in F^{(n)}-\left\{X\left(\sigma\left(F^{(n)}\right)\right)\right\}\right\} .
$$

In this section, we will obtain exponential bounds on $W \equiv W_{1}$ and using this bound we will deduce the upper bound for the transition density.

For $x \in F^{(n)} \cap E$, let $\Psi_{\omega^{1}}(E), \ldots, \Psi_{\omega^{m}}(E)\left(\left|\omega^{k}\right|=n\right.$ for $1 \leqq k \leqq m, m$ depends on $\left.x\right)$ be al the $n$-complexes containing $x$. For $s \geqq 0,1 \leqq i \leqq s_{0}$ and $1 \leqq j \leqq m$, set $B_{n, i, \omega^{j}}(x)=\left\{\omega: X\left(W_{n}\right) \in N_{n}^{i}(x) \cap \Psi_{\omega^{j}}(E)\right\}$ and define

$$
\phi_{\left(\omega^{1}, \ldots, \omega^{m}, \omega^{j}\right)}^{i}(s)=E^{x}\left[\exp \left(-s W_{n}\right) \mid B_{n, i, \omega^{j}}(x)\right] .
$$

Note that by Proposition $2.2(4), x$ is determined as the unique intersection of $\Psi_{\omega^{1}}(E), \ldots, \Psi_{\omega^{m}}(E)$.

Lemma 5.1. For all $s \geqq 0,1 \leqq k \leqq N$,

$$
\phi_{\left(\omega^{1} k, \ldots, \omega^{m} k ; \omega^{j} k\right)}^{i}(s)=\phi_{\left(k \omega^{1}, \ldots, k \omega^{m} ; k \omega^{J}\right)}^{i}(s)=\phi_{\left(\omega^{1}, \ldots, \omega^{m} ; \omega^{J}\right)}^{i}\left(\tau_{k}^{-1} s\right) .
$$

Proof. It is enough to show that $\tau_{k}$ is the time scaling factor on $\Psi_{k}(E)(1 \leqq k \leqq N)$. Using Proposition 2.8 and a well-known formula for point recurrent processes, we have

$$
E^{x}\left(e^{-\beta \sigma(\{y\})}\right)=\frac{g_{\beta}(x, y)}{g_{\beta}(y, y)}=\frac{\rho_{1} g_{\beta \tau_{1}}\left(\alpha_{1}^{-1} x, \alpha_{1}^{-1} y\right)}{\rho_{1} g_{\beta \tau_{1}}\left(\alpha_{1}^{-1} y, \alpha_{1}^{-1} y\right)}=E^{\alpha_{1}^{-1} x}\left(e^{-\beta \tau_{1} \sigma\left(\left\{\alpha_{1}^{-1} y\right\}\right)}\right),
$$

for all $x, y \in \hat{E}$. By this, we see that $\tau_{1}$ is a time scaling factor on $\Psi_{1}(E)$. For $k>1$, we first construct an unbounded fractal of which the origin is the fixed point of $\Psi_{k}(E)$. Then the Dirichlet form can be constructed using the scaling $\rho_{k}$. By the same argument as above, the hitting times of both diffusions are the same if the particle moves inside $E$. Thus we see that $\tau_{k}$ is a time scaling factor on $\Psi_{k}(E)$.

Proposition 5.2. Define $\gamma=\gamma^{\prime} /(S+1)$. Then, there exist $c_{5.1}, c_{5.2 . i}>0$ such that

$$
\phi_{\left(i_{1}, \ldots, i_{m}, i_{j}\right)}^{i}(s) \leqq c_{5.1} \exp \left(-c_{5.2 . i} \tau_{i_{j}}^{-\gamma} s^{\gamma}\right), \quad \forall s \geqq 0 .
$$

Proof. Set $\max _{i} \tau_{i}^{-1}=\alpha$ and $\min _{i} \tau_{i}^{-1}=\beta$. Take an arbitrary $c_{1} \in(0,1)$ and fix it. Because $\phi_{\left(i_{1}, \ldots, i_{m} ; i_{j}\right)}^{i}(s) \rightarrow 0$ as $s \rightarrow \infty$, it is possible to take a sufficiently small $\delta>0$ and sufficiently large $c_{2}>0$ such that

$$
\phi_{\left(i_{1}, \ldots, i_{m} ; i_{j}\right)}^{i}(s) \leqq c_{1} \exp \left(-\delta \tau_{i_{j}}^{-\gamma} \mathbf{x}_{i} s^{\gamma}\right) \text { for } s \in\left[c_{2} \alpha, c_{2}\right] .
$$

Here $\mathbf{x}_{i}$ is an $i$-th element of $\mathbf{x}>0$ whose existence was proved in Proposition 3.1. Now, define $\pi_{2}\left(x, N_{1}^{i}(x) \cap \Psi_{i_{J}}(E)\right)=\left\{\pi_{2}: \pi_{2}\right.$ is a 2 -walk from $x$ to an element of $N_{1}^{i}(x) \cap \Psi_{i_{j}}(E)$ which does not pass $\left.F^{(1)} \backslash\{x\}\right\}$. 
Also, for $F^{(2)}$-neighborhoods $p, q$, define

$$
\phi_{p, q}(s)=E^{p}\left(\exp \left(-s W_{2}\right) \mid X\left(W_{2}\right)=q\right) .
$$

Then by the strong Markov property,

Set

$$
\phi_{\left(i_{1}, \ldots, i_{m} ; i_{j}\right)}^{i}(s)=\sum_{\substack{\pi \in \pi_{2}\left(x, N_{1}^{i}(x) \cap \Psi_{l_{j}}(E)\right), \pi=\left\{p_{k}, p_{k+1}\right\}_{k=1}^{|\pi|}}} P^{x}\left[\pi \mid B_{1, i, i_{j}}(x)\right] \cdot \Pi_{k=1}^{|\pi|} \phi_{p_{k}, p_{k+1}}(s) .
$$

$\hat{\pi}_{2}=\left\{\pi \in \pi_{2} \mid \pi\right.$ is minimal with respect to the order introduced in Remark 3.3. $\}$

Then, noting that

$$
\sum_{\pi \in \pi_{2}} P^{x}\left[\pi \mid B_{1, i, i_{j}}(x)\right]=1
$$

and $0<\phi_{p_{k}, p_{k+1}}(s) \leqq 1$, we see from (5.2) that

$$
\phi_{\left(i_{1}, \ldots, i_{m} ; i_{j}\right)}^{i_{1}}(s) \leqq \sum_{\pi \in \hat{\pi}_{2}} \Pi_{k=1}^{|\pi|} \phi_{p_{k}, p_{k+1}}(s) .
$$

On the other hand each $\phi_{p_{k}, p_{k+1}}(s)$ is of the form

$$
\phi_{\left(i_{1}, \ldots, i_{m} ; i_{j}\right)}^{l^{\prime}}(s)=\phi_{\left(i_{1}, \ldots, i_{m} ; i_{j}\right)}^{l^{\prime}}\left(\tau_{1}^{-1} s\right)
$$

if $k=1$ (as the weight of 1 -complexes containing an element of $F^{(0)}$ is $r_{1}$ ), and

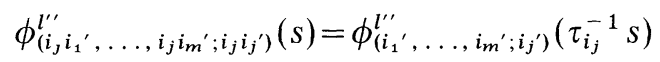

if $k>1$ (as the 2-complex containing $p_{k}$ is contained in $\Psi_{i_{j}}(E)$ ). Here the equalities hold because of Lemma 5.1. Now using (5.1) in (5.3), for $s \in\left[c_{2}, c_{2} / \beta\right]$ we have

$$
\phi_{\left(i_{1}, \ldots, i_{m}, i_{j}\right)}^{i_{1}}(s) \leqq \sum_{\pi \in \hat{\pi}_{2}} c_{1}^{|\pi|} \exp \left(-\delta \tau_{i_{j}}^{-\gamma}(G(\mathbf{x}))_{i} s^{\gamma}\right) .
$$

Choose $c_{1} \in(0,1)$ so that $\sum_{\pi \in \hat{\pi}_{2}} c_{1}^{|\pi|} \leqq c_{1}$, which is possible because $|\pi| \geqq 2$ for $\pi \in \hat{\pi}_{2}$. Then (5.2) holds for $s \in\left[c_{2}, c_{2} / \beta\right]$, too. Inductively, it follows that (5.1) holds for $s \in\left[\alpha c_{2}, \infty\right)$. As $\phi_{\left(i_{1}, \ldots, i_{m} ; i_{j}\right)}^{i}(s) \leqq 1$, retaking $c_{1}=\exp \left(\delta \tau_{r_{r_{j}}}^{-\gamma} \mathbf{x}_{i} c_{2}^{\gamma}\right)$ we have the required estimate.

Set

$$
W_{\Lambda_{n}}=\inf \left\{t \geqq 0: X(t) \in H^{\left(\Lambda_{n}\right)}-\left\{X\left(\sigma\left(H^{\left(\Lambda_{n}\right)}\right)\right)\right\}\right\} .
$$

Remark 5.3. From this point we consider unbounded affine nested fractals.

Theorem 5.4. There exist $c_{5.3}, c_{5.4}>0$ such that

$$
E^{x}\left(\exp \left(-s W_{\Lambda_{n}}\right)\right) \leqq c_{5.3} \exp \left(-c_{5.4}\left\{\rho_{i_{0}}^{-n(S+1)} s\right\}^{\gamma}\right) \forall x \in H^{\left(\Lambda_{n}\right)}
$$

Proof. First, we consider E. Let $x \in H^{\left(\Lambda_{n}\right)} \cap E$ be a boundary point of the $l \Lambda_{n}$ complexes $F_{i_{1}}, \ldots, F_{i_{1}}$. Without loss of generality, we can let the weights of $F_{i_{k}}$ be $r_{i_{1}}, \ldots, i_{m} r_{j_{k}} \underbrace{r_{1} \ldots r_{1}}$ for $1 \leqq k \leqq l$. Set $\max _{i=1}^{l} n(i)=u$. Then, $n(k)$ times

$$
\begin{aligned}
E^{x}\left(\exp \left(-s W_{\Lambda_{n}}\right)\right) & \leqq E^{x}\left(\exp \left(-s W_{m+1+u}\right)\right) \\
& \leqq \max _{k=1}^{l} \phi_{\left(j_{1}, \ldots, j_{l} j_{k}\right)}\left(\left(\tau_{i_{1}} \ldots \tau_{i_{m}} \tau_{1}^{u}\right)^{-1} s\right) \\
& \leqq c_{1} \exp \left(-c_{2}\left\{\left(\tau_{i_{1}} \ldots \tau_{i_{m}} \tau_{1}^{u}\right)^{-1} s\right\}^{\gamma}\right) \\
& \leqq c_{1} \exp \left(-c_{3}\left(\rho_{i_{0}}^{-n(S+1)} s\right)^{\gamma}\right)
\end{aligned}
$$


Here the second inequality is by Lemma 5.1, the third inequality is by Proposition 5.2 and the last inequality is by the fact that $\rho_{i_{0}}^{n} \leqq \rho_{i_{1}} \ldots \rho_{i_{m}} \rho_{1}^{u} \cdot \max _{i=1}^{N} \rho_{i}$. For $x \in H^{\left(\Lambda_{n}\right)}$ in the unbounded affine nested fractal, take $k$ such that $\Psi_{1}^{(k)}(x) \in E$. Then, by the above estimate and time scaling property, we can easily obtain the desired estimate.

Using a Tauberian theorem for exponential type for oscillating functions ([15]), we obtain the following.

Corollary 5.5. For $x \in H^{\left(\Lambda_{n}\right)}$,

$$
P^{x}\left(W_{\Lambda_{n}} \leqq s\right) \leqq c_{5.5} \exp \left(-c_{5.6}\left\{\rho_{i_{0}}^{n(S+1)} s\right\}^{\left.-\frac{\gamma}{1-\gamma}\right)} .\right.
$$

Now we are ready to deduce the upper bound on the transition density. We define $d_{w}=1 / \gamma$.

Lemma 5.6. There exist $c_{5.7}, c_{5.8}>0$ such that,

$$
P^{x}\left(\sup _{s \leqq t} d\left(X_{s}, X_{0}\right) \geqq \delta\right) \leqq c_{5.7} \exp \left(-c_{5.8}\left(\delta^{d_{w} t^{-1}}\right)^{\frac{1}{d_{w}-1}}\right), \quad \forall x \in \hat{E} .
$$

Proof. Choose $n$ so that $2 c_{3.2} \rho_{i_{0}}^{-n \gamma^{\prime}} \leqq \delta \leqq 2 c_{3.2} \rho_{i_{0}}^{-(n-1) \gamma^{\prime}}$. Then,

$$
\begin{aligned}
P^{x}\left(\sup _{s \leqq t} d\left(X_{s}, X_{0}\right) \geqq \delta\right) & \leqq \max _{y \in N_{\Lambda_{n}}(x)} P^{y}\left(W_{\Lambda_{n}} \leqq t\right) \\
& \leqq c_{5.5} \exp \left(-c_{5.6}\left\{\rho_{i_{0}}^{n(S+1)} t\right\}^{-\frac{1}{d_{w^{-}}-1}}\right) \\
& \leqq c_{5.5} \exp \left(-c_{5.8}\left\{\delta^{d_{w}} t^{-1}\right\}^{\frac{1}{d_{w^{-}}-1}}\right)
\end{aligned}
$$

Theorem 5.7. There exist $c_{5.9}, c_{5.10}>0$ such that,

$$
\hat{p}_{t}(x, y) \leqq c_{5.9} t^{-d_{s} / 2} \exp \left(-c_{5.10}\left(\frac{d(x, y)^{d_{w}}}{t}\right)^{\frac{1}{d_{w}-1}}\right), \quad \forall x, y \in \hat{E}, \forall t \in(0, \infty) .
$$

Proof. The following proof is the same as that of [4]. Fix $x \neq y$ and $t$ and let $\varepsilon<\frac{1}{6} d(x, y)$. For $a \in \hat{E}$, set $B_{\varepsilon}(a)=\{b \in \hat{E} \mid d(a, b)<\varepsilon\}$. Let $v_{x}=\left.\mu\right|_{B_{\varepsilon}(x)}, v_{y}=\left.\mu\right|_{B_{\varepsilon}(y)}$, $A_{1}=\left\{z \in \hat{E}: d(z, x) \leqq \frac{1}{2} d(x, y)\right\}, A_{2}=\hat{E}-A_{1}$ and $S=\inf \left\{t: d\left(X_{t}, X_{0}\right)>\frac{1}{3} d(x, y)\right\}$. Then

$$
\begin{aligned}
P^{v_{x}}\left(X_{t} \in B_{\varepsilon}(y)\right)= & P^{v_{x}}\left(X_{t} \in B_{\varepsilon}(y), X_{\frac{t}{2}} \in A_{1}\right) \\
& +P^{v_{x}}\left(X_{t} \in B_{\varepsilon}(y), X_{\frac{t}{2}} \in A_{2}\right) \equiv I_{1}+I_{2} .
\end{aligned}
$$

For $z \in B_{\varepsilon}(x)$, by Lemma 5.6,

$$
\begin{aligned}
P^{v_{x}}\left(X_{\frac{t}{2}} \in A_{2}\right) & \leqq P^{z}\left(S<\frac{t}{2}\right) \\
& \leqq c_{5.7} \exp \left(-c_{1}\left(\frac{d(x, y)^{d_{w}}}{t}\right)^{\frac{1}{d_{w}-1}}\right),
\end{aligned}
$$


while if $q(z) \equiv P\left(X_{t} \in B_{\varepsilon}(y) \mid X_{\frac{t}{2}}=z\right)$, then by Theorem 4.5,

$$
q(z)=\int_{B_{\varepsilon}(y)} \hat{p}_{\frac{t}{2}}(z, w) \mu(d w) \leqq c_{2} t^{-\frac{d_{s}}{2}} \mu\left(B_{\varepsilon}(y)\right) .
$$

Thus

$$
\begin{aligned}
I_{2} & =E^{v_{x}}\left(q\left(X_{\frac{t}{2}}\right): X_{\frac{t}{2}} \in A_{2}\right) \\
& \leqq c_{3} \mu\left(B_{\varepsilon}(x)\right) \mu\left(B_{\varepsilon}(y)\right) t^{-d_{s} / 2} \exp \left(-c_{1}\left(\frac{d(x, y)^{d_{w}}}{t}\right)^{\frac{t}{d_{w}-1}}\right) .
\end{aligned}
$$

For $I_{1}$, by the symmetry of $\hat{p}_{t}(x, y)$,

$$
P^{v_{x}}\left(X_{t} \in B_{\varepsilon}(y), X_{\frac{t}{2}} \in A_{1}\right)=P^{v_{y}}\left(X_{t} \in B_{\varepsilon}(x), X_{\frac{t}{2}} \in A_{1}\right) .
$$

which is bounded in exactly the same way as $I_{2}$.

Adding the bounds for $I_{1}$ and $I_{2}$,

$$
P^{v_{x}}\left(X_{t} \in B_{\varepsilon}(y)\right) \leqq 2 c_{3} \mu\left(B_{\varepsilon}(x)\right) \mu\left(B_{\varepsilon}(y)\right) t^{-d_{s} / 2} \exp \left(-c_{1}\left(\frac{d(x, y)^{d_{w}}}{t}\right)^{\frac{1}{d_{w}-1}}\right) .
$$

Dividing both sides by $\mu\left(B_{\varepsilon}(x)\right), \mu\left(B_{\varepsilon}(y)\right)$ and using the continuity of $\hat{p}_{t}(x, y)$ in $(x, y)$ proves the theorem.

For the transition density of the compact fractal, we have the following estimate by essentially the same proof.

Theorem 5.8. For sufficiently small $\delta>0$, there exist constants $c_{5.11}(\delta), c_{5.12}(\delta)$ such that

$$
p_{t}^{E}(x, y) \leqq c_{5.11}(\delta) t^{-d_{s} / 2} \exp \left(-c_{5.12}(\delta)\left(\frac{d(x, y)^{d_{w}}}{t}\right)^{\frac{1}{d_{w}-1}}\right) .
$$

for all $0<t \leqq 1, x, y \in B_{\delta}(0)$.

\section{Lower Bounds for the Heat Kernel}

In the previous section we obtained an upper bound for the heat kernel for affine nested fractals. The object of this section will be to obtain a corresponding lower bound. The approach is to use the scaling in the transition density and a Sobolev type inequality. We will work with the unbounded fractal as it has an exact scaling property. At the end we will need to prove a relationship between the transition density for the unbounded fractal and for the compact version. Our result can be stated as follows.

Theorem 6.1. There exists $c_{6.1}, c_{6.2}>0$ such that,

$$
\hat{p}_{t}(x, y) \geqq c_{6.1} t^{-d_{s} / 2} \exp \left(-c_{6.2}\left(\frac{d(x, y)^{d_{w}}}{t}\right)^{\frac{1}{d_{w}-1}}\right), \quad \forall x, y \in \hat{E}, \forall t \in(0, \infty) .
$$

The proof will follow from a series of lemmas with the main motivation for the approach coming from [4]. However we do not use any resolvent estimates, just the Dirichlet form and its scaling. 
Theorem 6.2. There exist constants $c_{6.3}, c_{6.4}$ such that

$$
\hat{p}_{t}(x, y) \geqq c_{6.3}, \quad \text { if } d(x, y)<c_{6.4}, \quad t \in\left[\tau_{1}^{-1}, 1\right] .
$$

The proof will follow from a series of lemmas. Firstly we control the diagonal of the transition density.

Lemma 6.3. There exists a constant $c_{6.5}$ such that

$$
\hat{p}_{t}(x, x) \geqq c_{6.5}, \quad \forall t \in\left[\tau_{1}^{-1}, 1\right], \forall x \in \hat{E} .
$$

Proof. This follows almost exactly the proof of [4] Lemma 7.1.

Lemma 6.4. Let $D_{n}$ be any $\Lambda_{n}$-complex. Then, there exists a constant $c_{6.6}$ such that

$$
\sup _{t \in\left[\tau_{1}^{-1}, 1\right]} \sup _{x, x^{\prime} \in D_{n}} \sup _{y \in E}\left|\hat{p}_{t}(x, y)-\hat{p}_{t}\left(x^{\prime}, y\right)\right| \leqq c_{6.6} \rho_{i_{0}}^{-n / 2} .
$$

Proof. As $\hat{p}_{t}(\cdot, y) \in \mathscr{F}$ we use Corollary 2.11. Noting that $D_{n}$ is a $\Lambda_{n}$-complex, we have

$$
\sup _{x, x^{\prime} \in D_{n}}\left|\hat{p}_{t}(x, y)-\hat{p}_{t}\left(x^{\prime}, y\right)\right| \leqq c_{1} \rho_{i_{0}}^{-n / 2} \hat{\mathscr{E}}\left(\hat{p}_{t}(\cdot, y), \hat{p}_{t}(\cdot, y)\right)^{1 / 2}
$$

Let $u(x)=\hat{p}_{t / 2}(x, y)$, so that $\hat{P}_{t / 2} u(x)=\hat{p}_{t}(x, y)$ and use [11] Lemma 1.3.3(i),

$$
\begin{aligned}
\widehat{\mathscr{E}}\left(\hat{P}_{t / 2} u, \hat{P}_{t / 2} u\right) & \leqq \frac{1}{t}\left(\|u\|_{2}^{2}-\left\|\hat{P}_{t / 2} u\right\|_{2}^{2}\right) \\
& \leqq \frac{1}{t} \hat{p}_{t}(y, y) \\
& \leqq \frac{1}{t} c_{4.5} t^{-d_{s} / 2} .
\end{aligned}
$$

Thus for $t \in\left[\tau_{1}^{-1}, 1\right]$ there is a $c_{6.6}$ such that

$$
\sup _{t \in\left[\tau_{1}^{-1}, 1\right]} \sup _{x, x^{\prime} \in D_{n}}\left|\hat{p}_{t}(x, y)-\hat{p}_{t}\left(x^{\prime}, y\right)\right| \leqq c_{6.6} \rho_{i_{0}}^{-n / 2} \text {. }
$$

Finaly observe that the constants obtained are independent of $y$.

Proof of Theorem 6.2. The lemmas above give the proof as for $x, y \in D_{n}$,

$$
\hat{p}_{t}(x, x) \geqq \hat{p}_{t}(x, x)-\left|\hat{p}_{t}(x, x)-\hat{p}_{t}(x, y)\right| \geqq c_{6.5}-c_{6.6} \rho_{i_{0}}^{-n / 2} .
$$

Hence we can choose a $c_{6.4}$ such that if $d(x, y) \leqq c_{6.4}$ then $x, y \in D_{n}$ for $n$ (or $\exists z \in H^{\left(\Lambda_{n}\right)}$ so that $x, z \in D_{n}$ and $\left.y, z \in D_{n}^{\prime}\right)$ and $c_{6.5}-c_{6.6} \rho_{i_{0}}^{-n / 2} \geqq c_{6.3}$.

This is enough to give the lower bound off the diagonal via the scaling in the transition density.

Corollary 6.5. There exist constants $c_{6.7}, c_{6.8}$ such that

$$
\hat{p}_{t}(x, y) \geqq c_{6.7} t^{-d_{s} / 2}, \quad \forall x, y: d(x, y)<c_{6.8} t^{1 / d_{w}}, \quad t \in(0,1] .
$$

Proof. By the scaling for the transition density and the theorem we can iterate the result from that on the interval $\left[\tau_{1}^{-1}, 1\right]$. If $t<\tau_{1}^{-1}$, let $n$ be such that $\tau_{1}^{n} t \in\left[\tau_{1}^{-1}, 1\right]$. 
Then, for $x, y$ such that $d(x, y)<c_{6.4} t^{1 / d w}<c_{6.4} \tau_{1}^{-n \gamma}$, by the scaling property of the distance (3.7), $d\left(\alpha_{1}^{n} x, \alpha_{1}^{n} y\right)<c_{6.4} \rho_{1}^{n \gamma} \tau_{1}^{-n \gamma} \leqq c_{6.4}$. Thus,

$$
\begin{aligned}
\hat{p}_{t}(x, y) & =\mu_{1}^{-n} \hat{p}_{\tau_{1}^{n} t}\left(\alpha_{1}^{n} x, \alpha_{1}^{n} y\right) \\
& \geqq c_{1} \mu_{1}^{-n}\left(\tau_{1}^{n} t\right)^{-d_{s} / 2} \\
& =c_{1} t^{-d_{s} / 2} .
\end{aligned}
$$

The final step is to use Aronson's method, a chaining argument, to extend the estimate from a small neighbourhood of the diagonal to all the off diagonal terms. For this purpose, we prepare some lemmas.

For the $\Lambda$-approximation to $\hat{E}$, we define $\pi_{\Lambda_{m}}, S_{\Lambda_{m}}^{i}$ and $G^{\Lambda_{m}}(\mathbf{x})$ in the same way as those defined in (3.1), (3.2) and (3.3), but with respect to $H^{\left(\Lambda_{m}\right)} \cap E$ and with respect to $\Lambda_{m}$-walk. Here $\pi=\left\{\left(q_{k}, q_{k+1}\right)\right\}_{k=1}^{l}$ is called a $\Lambda_{m}$-walk if $l \in \mathbf{N}, q_{k} \in H^{\left(\Lambda_{m}\right)}$ for $1 \leqq k \leqq l, q_{k}$ and $q_{k+1}$ join in the same $\Lambda_{m}$-cell for $1 \leqq k \leqq l-1$. For $\tilde{\rho}_{i}=\lambda / \tilde{r}_{i}$ $\left(1 \leqq i \leqq k_{0}\right)$, define

$$
\tilde{\Lambda}_{n}=\left\{\omega=\omega_{1} \ldots \omega_{m} \mid \tilde{\rho}_{\omega_{1} \ldots \omega_{m-1}}<\rho_{i_{0}}^{n} \leqq \tilde{\rho}_{\omega_{1} \ldots \omega_{m}}, 1 \leqq \omega_{i} \leqq k_{0}(1 \leqq i \leqq m)\right\} .
$$

Then we have the following.

Proposition 6.6. Let $A \in K_{\gamma^{\prime}}\left(K_{\gamma^{\prime}}\right.$ is defined in (3.4)), satisfying $G_{\gamma^{\prime}}(\mathbf{x})=\mathbf{x}$ so that it has the decomposition $A=\sum_{k} \tilde{\rho}_{k}^{-\gamma^{\prime}} A_{k}$ as in the proof of Proposition 3.1. Then, for all $n \geqq 1, \mathrm{G}^{\Lambda_{n}}(\mathbf{x})=\mathbf{x}$ and $A^{\left(\Lambda_{n}\right)} \equiv \sum_{\omega \in \tilde{\Lambda}_{n}} \tilde{\rho}_{\omega}^{-\gamma^{\prime}} A_{\omega}\left(A_{\omega}=A_{\omega_{1} \ldots \omega_{|\omega|}}\right)$ attains the minimum in $G^{\Lambda_{n}}$.

Proof. Let $G^{\Lambda_{n}}(\mathbf{x})=\left(\min _{\mathbf{a} \in S_{\Lambda_{m}}^{i}} \mathbf{a} \cdot \mathbf{x}\right)_{1 \leqq i \leqq s_{0}}=A_{\Lambda_{n}} \mathbf{x}$ and $\max _{\omega \in \tilde{A}_{n}}|\omega|=\bar{m}(|\omega|$ is the length of the word $\omega$ ) where $A_{A_{n}}$ is an $s_{0} \times s_{0}$-matrix so that the $i$-th row belongs to $S_{\Lambda_{m}}^{i}\left(1 \leqq i \leqq S_{0}\right)$. Then, by (3.8) and (3.9), if $\omega=\omega_{1} \ldots \omega_{\bar{m}} \in \tilde{\Lambda}_{n}$ then $\omega_{1 \ldots} \omega_{\bar{m}-1} i \in \tilde{\Lambda}_{n}$ for all $1 \leqq i \leqq k_{0}$. By the symmetry and the self-similarity of the fractal, we can decompose $A_{\Lambda_{n}}$ into $\sum_{\omega \in \tilde{X}_{n},|\omega|<\bar{m}} \tilde{\rho}_{\omega}^{-\gamma^{\prime}} A(\omega)+\sum_{\omega \in \tilde{X}_{n},|\omega|=\bar{m}} \tilde{\rho}_{\omega-1}^{-\gamma^{\prime}} A(\omega-1) B(\omega)$ for some $k_{0} \times k_{0}$-matrices $A(\omega), A(\omega-1)$ and $B(\omega) \in K$ (here $\omega-1=\omega_{1} \ldots \omega_{\bar{m}-1}$ for $\left.\omega=\omega_{1} \ldots \omega_{\bar{m}}\right)$. Iterating this decomposition, we have $A_{\Lambda_{n}}=\sum_{\omega \in \tilde{A}_{n}} B_{\omega}$, where $B_{\omega}=B\left(\omega_{1}\right) B\left(\omega_{1} \omega_{2}\right) \ldots B(\omega)\left(B\left(\omega_{1} \ldots \omega_{i}\right) \in K, 1 \leqq i \leqq|\omega|\right)$. On the other hand, as $B(\omega) \in K, B(\omega) \mathbf{x} \geqq \mathbf{x}$ and so $A_{\Lambda_{n}} \mathbf{x} \geqq \mathbf{x}$. As $A^{\left(\Lambda_{n}\right)} \mathbf{x}=\mathbf{x}$, by definition of $G^{\Lambda_{n}}(\mathbf{x}), A^{\left(\Lambda_{n}\right)}$ attains the minimum.

Lemma 6.7. There exists $c_{6.8}>0$ which satisfies the following for all $x, y \in \hat{E}, m \in \mathbf{Z}$ and $k \in \mathbf{N}$ :

If $d(x, y)<\rho_{i_{0}}^{-m \gamma^{\prime}}$, then there exist $x_{0}, x_{1}, \ldots, x_{n}\left(c_{6.9} \rho_{i_{0}}^{k \gamma^{\prime}} \leqq n \leqq c_{6.10} \rho_{i_{0}}^{k \gamma^{\prime}}\right)$ such that $x_{0}=x, x_{n}=y, x_{1}, \ldots, x_{n-1} \in H^{\left(\Lambda_{m+k}\right)}$ and $x_{i}, x_{i+1}$ lie in the same $\Lambda_{m+k}$-complex for $0 \leqq i \leqq n-1$.

Proof. For $x, y \in H^{\left(\Lambda_{n}\right)}$, set

$$
\begin{gathered}
\tilde{d}_{\Lambda_{n}}(x, y)=\min \left\{|\pi|: \pi \in \pi_{\Lambda_{n}}(x, y)\right\}, \\
L_{n}=\max _{x, y \in F^{(0)} \cap E} \tilde{d}_{\Lambda_{n}}(x, y) .
\end{gathered}
$$

Firstly we show that $L_{n} \leqq c_{1} \rho_{i_{0}}^{n \gamma^{\prime}}$ for some $c_{1}>0$. By the above proposition,

$$
G^{A_{n}}(\mathbf{x})=\sum_{\omega \in \tilde{\Lambda}_{n}} \tilde{\rho}_{\omega}^{-\gamma^{\prime}} A_{\omega} \mathbf{x}=\mathbf{x}
$$


Let

$$
\left(\begin{array}{c}
v_{1} \\
\vdots \\
v_{k_{0}}
\end{array}\right)=\sum_{\omega \in \tilde{\Lambda}_{n}} A_{\omega}\left(\begin{array}{l}
1 \\
\vdots \\
1
\end{array}\right) .
$$

Then $v_{i}$ is the number of steps for the $\Lambda_{n}$-walk leading from 0 to $N_{i}(0)$. As $\rho_{\omega-1}<\rho_{i_{0}}^{n} \leqq \rho_{\omega}$ and $\mathbf{x}>0$, we see that $\max _{i} v_{i} \leqq c_{1} \rho_{i_{0}}^{n \gamma^{\prime}}$ for some $c_{1}>0$, thus $L_{n} \leqq c_{1} \rho_{i_{0}}^{n \gamma^{\prime}}$.

Next, we show that

$$
\limsup _{n \rightarrow \infty} \max _{x \in H^{\left(\Lambda^{\prime}\right.} \cap E} \max _{y \in F^{(0)} \cap E} \frac{\tilde{d}_{\Lambda_{n}}(x, y)}{\rho_{i_{0}}^{n \gamma^{\prime}}}<\infty .
$$

Set $q=\max _{x \in H^{\left(\Lambda_{2}\right)} \cap E} \max _{y \in F^{(0)} \cap E} \tilde{d}_{\Lambda_{2}}(x, y)$. Then, we have

$$
\begin{aligned}
\max _{x \in H^{\left(\Lambda_{n}\right)} \cap E} \max _{y \in F^{(0)} \cap E} \frac{\tilde{d}_{\Lambda_{n}}(x, y)}{\rho_{i_{0}}^{n \gamma^{\prime}}} & \leqq q \sum_{k=1}^{n-1} \frac{L_{k+1}}{\rho_{i_{0}}^{n \gamma^{\prime}}}+\frac{q}{\rho_{i_{0}}^{n \gamma^{\prime}}} \\
& \leqq q c_{1} \sum_{k=0}^{n-2} \frac{1}{\rho_{i_{0}}^{k \gamma^{\prime}}}+\frac{q}{\rho_{i_{0}}^{n \gamma^{\prime}}} \\
& \leqq \frac{2 q c_{1}}{\rho_{i_{0}}^{\gamma^{\prime}}-1} .
\end{aligned}
$$

Now, if $d(x, y)<\rho_{i_{0}}^{-m \gamma^{\prime}}$, then there exists $z \in H^{\left(\Lambda_{m-1}\right)}$ such that each pair $x, z$ and $z, y$ lies in the same $\Lambda_{m-1}$-complex. Take $x^{\prime} \in H^{\left(\Lambda_{m+k}\right)}$ which lies in the same $\Lambda_{m+k}$-complex as $x$ and also take $y^{\prime}$ in the same way. Then, by the self-similarity of the fractal and by the fact we have proved above, we know that we can take a sequence of the elements of $H^{\left(\Lambda_{m+k}\right)}$ which connects $x^{\prime}$ and $y^{\prime}$ with at most $c_{2} \rho_{i_{0}}^{-k \gamma^{\prime}}$ points for some large $c_{2}$. By moving at the same points of $H^{\left(\Lambda_{n}\right)}$ if necessary, we can take the length of the sequence greater than $c_{3} \rho_{i_{0}}^{k \gamma^{\prime}}$ for $c_{3}<c_{2}$.

Proof of Theorem 6.1. The idea of the proof is just the same as [4] and [23].

Let $L=d(x, y)^{d_{w} / t}$. By Corollary 6.5 , the theorem is already proved if $L \leqq c_{6.8}$. Thus we assume $L \geqq c_{6.8}$. For arbitrarily fixed $c_{1}>0$, choose $n$ and $m$ so that

$$
\begin{gathered}
\rho_{i_{0}}^{-n(s+1)(\gamma-1)}>c_{1} L \geqq \rho_{i_{0}}^{-(n+1)(s+1)(\gamma-1)}, \\
\rho_{i_{0}}^{-m(s+1) \gamma}>d(x, y) \geqq \rho_{i_{0}}^{-(m+1)(s+1) \gamma} .
\end{gathered}
$$

By the above lemma, we can pick the sequence $x_{0}, x_{1}, \ldots, x_{v}$ $\left(c_{6.9} \rho_{i_{0}}^{n \gamma^{\prime}} \leqq v \leqq c_{6.10} \rho_{i_{0}}^{n \gamma^{\prime}}\right)$ such that $x_{0}=x, x_{v}=y, x_{1}, \ldots, x_{v-1} \in H^{\left(\Lambda_{m+n}\right)}$ and $x_{i}, x_{i+1}$ lie in the same $\Lambda_{m+n}$-complex for $0 \leqq i \leqq v-1$. Let $B_{i}$ be the union of the $\Lambda_{n+m^{-}}$ complexes which contain $x_{i}$. Note that if $z \in B_{i-1}$ and $z^{\prime} \in B_{i}$, then $d\left(z, z^{\prime}\right) \leqq 3 \rho_{i_{0}}^{-(m+n)(s+1) \gamma} \leqq c_{6.8}(t / v)^{1 / d_{w}}$, when $c_{1}$ is chosen small enough. Thus, by Corollary $6.5, \hat{p}\left(t / v, z, z^{\prime}\right) \geqq c_{6.7}(t / v)^{-d_{s} / 2}$. Therefore

$$
\begin{aligned}
\hat{p}(t, x, y) & \geqq \int_{B_{1}} \ldots \int_{B_{v-1}} \hat{p}\left(t / v, x, y_{1}\right) \ldots \hat{p}\left(t / v, y_{n-1}, y\right) d \mu\left(y_{1}\right) \ldots d \mu\left(y_{n-1}\right) \\
& \geqq\left(\Pi_{i=1}^{v-1} \mu\left(B_{i}\right)\right) c_{6.7}^{v}(t / v)^{-d_{s} v / 2} \\
& \geqq c_{6.7}^{v}\left\{\Pi_{i=1}^{v-1}\left(\mu\left(B_{i}\right)(t / v)^{-d_{s} / 2}\right)\right\}(t / v)^{-d_{s} / 2} .
\end{aligned}
$$


As $\rho_{i_{0}}^{-(m+n) S} \leqq \mu\left(B_{i}\right) \leqq c_{2} \rho_{i_{0}}^{-(m+n) S}$ and $c_{6.9} \rho_{i_{0}}^{n \gamma^{\prime}} \leqq v \leqq c_{6.10} \rho_{i_{0}}^{n \gamma^{\prime}}$, from (6.2), (6.3) we see that $\mu\left(B_{i}\right)(t / v)^{-d_{s} / 2}$ is bounded above and below by positive constants which are independent of $L$ and $t$. Thus we have

$$
\begin{aligned}
\hat{p}(t, x, y) & \geqq c_{3}^{v} c_{4}(t / v)^{-d_{s} / 2} \\
& \geqq c_{3}^{v} c_{4} t^{-d_{s} / 2} \\
& \geqq c_{4} t^{-d_{s} / 2} \exp \left(-c_{6.10} \rho_{i_{0}}^{n \gamma^{\prime}} \log c_{3}^{-1}\right) .
\end{aligned}
$$

Substituting our choice in the last term completes the proof.

Following [5], we can derive the bound for the compact fractal from this result.

Theorem 6.8. For sufficiently small $\delta>0$ there exist constants $c_{6.11}(\delta), c_{6.12}(\delta)$ such that

$$
p_{t}^{E}(x, y) \geqq c_{6.11}(\delta) t^{-d_{s} / 2} \exp \left(-c_{6.12}(\delta)\left(\frac{d(x, y)^{d_{w}}}{t}\right)^{\frac{1}{d_{w}-1}}\right)
$$

for all $0<t \leqq 1, x, y \in B_{\delta}(0)$.

Proof. It is easy to see that the inequality

$$
p_{t}^{E}(x, y) \geqq p_{t}^{a}(x, y)
$$

holds between the transition densities for the reflected process and the process absorbed at the boundary of $E$. We can now write the transition density for the absorbed process in terms of the transition density for the process on the unbounded fractal as

$$
p_{t}^{a}(x, y)=\hat{p}_{t}(x, y)-E\left(I_{\{\sigma(\hat{r} E) \leqq t\}} \hat{p}_{t-\sigma(\hat{r} E)}\left(X_{\sigma(\hat{r} E)}, y\right)\right) .
$$

Then, following [5] Theorem 7.11, we can obtain the result for $p_{t}^{a}(x, y)$ and hence for $p_{t}^{E}(x, y)$.

Remark. In the case of the Sierpinski gasket it is possible to map the path on the bounded gasket into the unit triangle by a succession of folds about the central triangle. This mapping is constructed by hand in [30]. Then the point $y \in E$ is the image of an infinite number of points in $\hat{E}$, and thus we can express the transition density as

$$
p_{t}^{E}(x, y)=\hat{p}_{t}(x, y)+\sum_{y^{\prime}} \hat{p}_{t}\left(x, y^{\prime}\right) .
$$

The result is just the first term.

Acknowledgement. We thank Prof. M. T. Barlow for fruitful discussions. Also, the third author thanks Dr. T. Kazumi for kind advice in Sect. 6 and Dr. J. Kigami for informing him of his new results.

\section{References}

1. Bandt, C., Stahnke, J.: Self-similar sets 6: Interior distance on deterministic fractals. Preprint, 1991

2. Barlow, M.T.: Random walks, electrical resistance, and nested fractals. In: K. D. Elworthy, N. Ikeda (eds.) Asymptotic Problems in Probability Theory: Stochastic models and diffusions on fractals. Pitman: Montreal: 1993, pp. 131-157 
3. Barlow, M.T., Bass, R.F.: Construction of Brownian motion on the Sierpinski carpet. Ann Inst H. Poincaré 25, 225-257 (1989)

4. Barlow, M.T., Bass, R.F.: Transition densities for Brownian motion on the Sierpinski carpet. Probab. Theory Relat. Fields 91, 307-330 (1991)

5. Barlow, M.T., Perkins, E.A.: Brownian motion on the Sierpinski gasket. Probab. Theory Rel. Fields 79, 543-624 (1988)

6. Carlen, E.A., Kusuoka, S., Stroock, D.W.: Upper bounds for symmetric Markov transition functions. Ann. L'IHP, Sup au No. 2, 245-287 (1987)

7. Davies, E.B.: One-parameter semigroups. Cambridge: Cambridge University Press, 1980

8. Davies, E.B.: Heart kernels and spectral theory. Cambridge: Cambridge University Press, 1989

9. Dynkin, E.B.: Excessive measures and harmonic functions for Markov processes. Trans. Am. Math. Soc. 258, 217-244 (1980)

10. Fujita, T.: Some asymptotic estimates of transition probability densities for generated diffusion processes with self-similar speed measures. Publ. Res. Inst. Math. Sci. 26, 819-840 (1990)

11. Fukushima, M.: Dirichlet forms and Markov processes. Amsterdam: North-Holland, 1980

12. Fukushima, M.: Dirichlet forms, diffusion processes and spectral dimensions for nested fractals. In: Albeverio, Fenstad, Holden and Lindstrøm (eds.), Ideas and Methods in Mathematical Analysis, Stochastics, and Applications. In Memory of R. Høegh-Krohn, vol. 1, Cambridge Univ. Press, 1992, pp. 151-161

13. Havlin, S, Ben-Avraham, D.: Diffusion in disordered media. Adv. Phys. 36, 695-798 (1987)

14. Hutchinson, S.: Self-similar sets. Indiana Univ. Math. J. 30, 713-747 (1981)

15. Kasahara, Y.: Tauberian theorems of exponential type. J. Math. Kyoto Univ. 18, 209-219 (1978)

16. Kigami, J.: On a harmonic calculus on the Sierpinski spaces. Japan J. Appl. Math. 6, 259-290 (1989)

17. Kigami, J.: Harmonic calculus on P.C.F. self-similar sets. Trans. Am. Math. Soc. 335, 721-755 (1993)

18. Kigami, J.: Hausdorff dimension of self-similar sets and shortest path metric. Preprint, 1992

19. Kigami, J.: Effective resistances for harmonic structures on P.C.F. self-similar sets. To appear in Math. Proc. Cam. Phil. Soc., 1993

20. Kigami, J., Lapidus, M.L.: Weyl's problem for the spectral distribution of Laplacians on P.C.F. self-similar fractals. Commun. Math. Phys. 158, 93-125 (1993)

21. Krebs, W.B.: Hitting time bounds for brownian motion on a fractal. Proc. Am. Math. Soc. 118, 223-232 (1993)

22. Kumagai, T.: Regularity, closedness and spectral dimensions of the Dirichlet forms on P.C.F. self-similar sets. J. Math. Kyoto Univ. 33, 765-786 (1993)

23. Kumagai, T.: Estimates of the transition densities for Brownian motion on nested fractals. Probab. Theory Relat. Fields 96, 205-224 (1993)

24. Kusuoka, S.: Dirichlet forms on fractals and products of random matrices. Publ. Res. Inst. Math. Sci. 25, 659-680 (1989)

25. Kusuoka, S.: Lecture on diffusion processes on nested fractals. To appear in Springer Lect. Notes in Math.

26. Kusuoka, S., Zhou, X.Y.: Dirichlet forms on fractals: Poincaré constant and resistance. Probab. Theory Relat. Fields 93, 169-196 (1992)

27. Lindstrøm, T.: Brownian motion on nested fractals. Memoris Am. Math. Soc. 420, 83 (1990)

28. Murai, J.: Personal communication

29. Okada, M., Sekiguchi, T., Shiota, Y.: Heat kernels on infinite graph networks and deformed Sierpinski gaskets. Japan J. Appl. Math. 7, 527-543 (1990)

30. Pietruska-Paluba, K.: The Lifschitz singularity for the density of states on the Sierpinski gasket. Probab. Theory Relat. Fields 89, 1-33 (1991)

31. Shima, T.: Lifschitz tails for random Schrödinger operators on nested fractals. Osaka J. Math. 29, 749-770 (1992) 